\title{
Role of type-specific neuron properties in a spinal cord motor network
}

\author{
Bart Sautois • Stephen R. Soffe • Wen-Chang Li • \\ Alan Roberts
}

Received: 7 July 2006 / Revised: 23 October 2006 / Accepted: 26 December 2006 / Published online: 20 January 2007

(C) Springer Science + Business Media, LLC 2007

\begin{abstract}
Recent recordings from spinal neurons in hatchling frog tadpoles allow their type-specific properties to be defined. Seven main types of neuron involved in the control of swimming have been characterized. To investigate the significance of type-specific properties, we build models of each neuron type and assemble them into a network using known connectivity between: sensory neurons, sensory pathway interneurons, central pattern generator (CPG) interneurons and motoneurons. A single stimulus to a sensory neuron initiates swimming where modelled neuronal and network activity parallels physiological activity. Substitution of firing properties between neuron types shows that those of excitatory CPG interneurons are critical for stable swimming. We suggest that type-specific neuronal properties can reflect the requirements for involvement in one particular network response (like swimming), but may also reflect the need to participate in more than one response (like swimming and slower struggling).
\end{abstract}

Keywords Tadpole model $\cdot$ Swimming $\cdot$ CPG . Type-specificity $\cdot$ NMDA

Action Editor: Eberhard E. Fetz

B. Sautois $(\square)$

Department of Applied Mathematics and Computer Science,

Ghent University,

Krijgslaan 281-S9,

B-9000 Ghent, Belgium

e-mail: bart.sautois@ugent.be

S. R. Soffe · W.-C. Li - A. Roberts

School of Biological Sciences, University of Bristol, Bristol, BS8 1UG UK

\section{Introduction}

In many neuronal circuits, in both invertebrates and vertebrates, different neurons have rather different properties. We would like to understand the significance of these typespecific differences. However, particularly in the complex vertebrate central nervous system, the normal activity of neurons during behaviour is difficult to study. To investigate the significance of different neuronal properties we need to investigate a region with different types of neuron where the normal activity of the neurons and the region are known. The spinal cord of vertebrates has a range of different neuron types with different properties. In some simpler cases like the lamprey (Parker, 2001; Grillner and Wallen, 2002), and the early developmental stages of the zebrafish (Higashijima et al., 2004a, b) and frog Xenopus (Roberts, 2000), there is information on the neuron types and their activity during swimming. In lamprey and Xenopus this has allowed modelling of the spinal networks underlying the generation of swimming activity and the testing of hypotheses on the neurons and cellular mechanisms responsible (e.g. Kotaleski et al., 1999; Tunstall et al., 2002). So far, while some modelling has introduced stochasticity (Hellgren et al., 1992), it has not addressed the significance of the different properties of the component neuron types, in part because detailed data were not available.

The newly hatched Xenopus tadpole, with only a couple of thousand neurons in the spinal cord, provides a suitable system to study the significance of type-specific differences between neurons. These neurons can be divided into 8 distinct classes on the basis of clear anatomical differences (in dorso-ventral soma position, dendrite distribution and axonal projections; Roberts, 2000; Li et al., 2001). Nothing is known about the function of one class of possible neural canal receptor neuron (Dale et al., 1987). For the remaining neuron 
classes, using single-cell recordings (including a new wholecell patch recording method (Li et al., 2004c), in the spinal cord of immobilized tadpoles, we have defined the typespecific properties of individual neurons whose anatomical features were revealed by neurobiotin injection. These include: their passive and active responses to current injection, the transmitter pharmacology of synapses they make and receive (where some synaptic conductances have been studied in voltage-clamp recordings), and their activity during responses to sensory stimuli. Such detailed information has shown that 7 of the anatomical classes correspond to distinct functional classes: 1 type of sensory neuron innervating the skin (Clarke et al., 1984; Li et al., 2003); 2 types of sensory pathway neuron that have a role in the initiation of swimming (Li et al., 2003, 2004b); 3 types of premotor neuron that are active during swimming and may be components of the swimming central pattern generator (Dale, 1985; Li et al., 2002, 2004d, 2006); and motoneurons (Soffe and Roberts, 1982; Roberts et al., 1999). These detailed studies have recently provided evidence of functional sub-types within two anatomical classes (Li et al., unpublished). We should emphasize that, apart from the primary sensory neurons (Ribera and Nguyen, 1993), there is presently no evidence on the ionic channels that different specific types of Xenopus spinal neurons possess.

Like the different locomotor gaits shown by mammals, the neuronal network in the young tadpole spinal cord is able to generate two rather different patterns of motor output. In response to brief touch to the skin, the tadpole swims using alternating lateral bends in the trunk that move in a wave from head to tail at frequencies from 10 to $25 \mathrm{~Hz}$ (Kahn et al., 1982). On the other hand, when tadpoles are held and the skin stimulated continuously, they struggle, making stronger, slower bends at 2 to $5 \mathrm{~Hz}$ that move from the tail to the head (Kahn and Roberts, 1982). In immobilized tadpoles, very different patterns of activity in spinal interneurons and motoneurons can be recorded during these two responses. During swimming spinal neurons fire a single action potential on each cycle but during struggling they fire high frequency bursts lasting nearly half a cycle (Soffe, 1993).

Our aim is to investigate the significance of type-specific properties of neurons in the operation of neuronal networks, using the spinal neurons and networks responsible for generating swimming activity in young tadpoles. Previous models of the hatchling Xenopus tadpole spinal swimming circuits have assumed that all CPG neurons have the same properties (Roberts and Tunstall, 1990; Dale, 1995b; Tunstall et al., 2002; Dale, 2003). Data from recent recordings show that this assumption is wrong: different neuron types show clearly distinct properties. This puts us in a nearly unique position for a vertebrate where we can make models of each individual type of neuron and their synapses on the basis of available physiological data. We then use these individual neuron models to construct a sensory network coupled to a motor pattern generating network. In response to a brief "sensory" stimulus, this network reliably produces the swimming behaviour typical for the young Xenopus tadpole. By using neurons with clearly distinct properties based on physiology, we were able to use realistic conductance strengths for the different synaptic connections in this network. By changing the properties of neurons in the network, we investigate the significance of the type-specific properties of the neurons in the generation of swimming activity. The simple networks used here are a first step towards more complex population models that can also generate the tadpole's struggling activity (Li et al., in prep).

\section{Methods}

\subsection{Physiological measurements}

Methods for recording neuron activity and responses have been described recently (Li et al., 2006). Briefly, Xenopus tadpoles at stage 37/38 (Nieuwkoop and Faber, 1956) were anaesthetised and then immobilized in $\alpha$-bungarotoxin. After this tadpoles were dissected to reveal neuron cell bodies while pinned onto a rotatable rubber platform in a bath of saline (concentrations in $\mathrm{mM}: \mathrm{NaCl} 115, \mathrm{KCl} 3, \mathrm{CaCl}_{2} 2$, $\mathrm{NaHCO}_{3} 2.4, \mathrm{MgCl}_{2} 1$, HEPES 10 , adjusted with $5 \mathrm{M} \mathrm{NaOH}$ to $\mathrm{pH}$ 7.4). Whole-cell patch electrodes contained $0.1 \%$ neurobiotin in an intracellular solution (concentrations in $\mathrm{mM}$ : K-gluconate 100, $\mathrm{MgCl}_{2}$ 2, EGTA 10, HEPES 10, $\mathrm{Na}_{2}$ ATP 3 , NaGTP 0.5 adjusted to $\mathrm{pH} 7.3$ with $\mathrm{KOH}$ ). Recordings from individual neurons were made under visual control using a $\times 40$ water immersion lens on an upright compound microscope. The methods for fixation, neurobiotin staining and recording of neuron anatomy have been described previously (Li et al., 2002). All experiments comply with UK Home Office regulations and have been approved following local ethical review.

\subsection{Modelling}

\subsubsection{Individual neurons}

To begin the modelling of individual neurons, we started from previous models built specifically for Xenopus spinal neurons (Roberts and Tunstall, 1990; Dale, 1995a, b). We used a single compartment model since these small neurons have very short dendrites and, if the axon is ignored, are electrically compact (Dale, 1995b; Wolf et al., 1998). Some parameters were taken directly from experimental data on individual neuron types (e.g. leak conductance and reversal potential). However, since membrane currents have not yet been characterized for the different neurons types, 
Table 1 Measured properties for the different neuron types based on whole-cell recordings (medians, based on 5-20 neurons of each type)

\begin{tabular}{|c|c|c|c|c|c|c|c|}
\hline & aIN & $\mathrm{MN}$ & $\mathrm{dIN}$ & $\mathrm{RB}$ & dlc & dla & $\mathrm{cIN}$ \\
\hline Input resistance $(\mathrm{M} \Omega)$ & 740 & 405 & 272 & 230 & 428 & 1436 & 206 \\
\hline Resting potential (mV) & -54 & -61 & -51 & -70 & -66 & -63 & -60 \\
\hline Current threshold for firing $(\mathrm{pA})$ & 28 & 109 & 85 & & 95 & 53 & 198 \\
\hline Action potential (AP) threshold (mV) & -29 & -26 & -28 & -25 & -35 & -32 & -25 \\
\hline AP peak $(\mathrm{mV})$ & 27 & 26 & 29 & 40 & 28 & 37 & 32 \\
\hline AP width (ms) & 1.8 & 0.9 & 1.9 & 1.5 & 0.5 & 0.75 & 0.8 \\
\hline Max. AP rise rate $(\mathrm{mV} / \mathrm{ms})$ & 121 & 105 & 85 & & 127 & 130 & 124 \\
\hline Max. AP fall rate (mV/ms) & 97 & 77 & 76 & & 137 & 100 & 101 \\
\hline Initial firing frequency $(\mathrm{Hz})$ & 24 & 41 & & & 97 & 29 & 71 \\
\hline Slope of frequency-current relationship $(\mathrm{Hz} / \mathrm{pA})$ & 53 & & & & 67 & & 84 \\
\hline Afterhyperpolarization (AHP) trough (mV) & -47 & -42 & -43 & & -44 & -57 & -43 \\
\hline AHP delay (ms) & 1.8 & 2.3 & 6.9 & & 1.3 & 2.1 & 1.5 \\
\hline Response to injected current & $\mathrm{RF}^{*}$ & $\mathrm{RF}^{*}$ & $\mathrm{~S}^{*}$ & $\mathrm{~S}^{*}$ & $\mathrm{AF}^{*}$ & $\mathrm{AF}^{*}$ & $\mathrm{DF}^{*}$ \\
\hline Initial gap before delayed burst (ms) & & & & & & & 167 \\
\hline Slope of gap-current relationship (ms/pA) & & & & & & & -200 \\
\hline
\end{tabular}

${ }^{*} \mathrm{RF}=$ Repetitive Firing, $\mathrm{S}=$ Single spike, $\mathrm{AF}=$ Adapting Firing, $\mathrm{DF}=$ Delayed Firing.

in most cases we used "emerging" parameters obtained by matching outputs of the model to physiological properties measured for each neuron type. Information was available from experiments on a wide range of these properties: neuronal input resistance, resting potential, firing threshold, current threshold for firing, action potential (AP) height and width, maximum rise and fall rates of the action potential, after-hyperpolarisation amplitude and delay, initial firing frequency to long, constant-current input, and the effect of increasing current on this frequency (see Table 1; Li et al., 2002, 2004b, d, 2006).

The general equations are based on Hodgkin and Huxley (1952). The differential equation for the voltage is:

$C \frac{d V}{d t}=I_{i n j}+I_{s y n}-$ Currents

$C$ represents the cell's membrane capacitance, $I_{i n j}$ represents experimentally injected current and $I_{s y n}$ the synaptic currents. Currents is the combination of all modelled ion channel currents. The number of these depends on the cell type, but all neurons have at least the following currents:

$$
\begin{aligned}
\text { Currents }= & g_{\text {leak }}\left(V-V_{\text {leak }}\right)+g_{N a} h_{N a} m_{N a}^{3}\left(V-V_{N a}\right) \\
& +g_{\text {Kfast }} n_{\text {Kfast }}\left(V-V_{\text {Kfast }}\right) \\
& +g_{\text {Kslow }} n_{\text {Kslow }}\left(V-V_{\text {Kslow }}\right)
\end{aligned}
$$

The currents include one leak current, one Na-current and two K-currents, one fast and one slow. The Na-current has both an activation and an inactivation component, while the K-currents only have the activating part (so for present purposes are assumed to be non-inactivating; cf Dale (1995b)).
The leak conductance $\left(g_{\text {leak }}\right)$ is the inverse of the measured input resistance (rescaled to nS), and the leak reversal potential $\left(V_{\text {leak }}\right)$ is given by the resting potential (clearly a mixed current, since in all neurons it was less negative than the reversal potential for potassium). The maximum conductances for sodium and potassium are based on Dale (Dale, 1995a, b), and matching to experimental results. In the absence of detailed measures, the neuronal input capacitance was assumed to be the same for all neurons and was set to $4 \mathrm{pF} . h_{N a}, m_{N a}$, $n_{\text {Kfast }}$ and $n_{\text {Kslow }}$ are gate variables defining the rate of opening and closing of specific membrane channels. Their behaviour is defined by the equation:

$\frac{d \text { gate }}{d t}=\frac{1}{t_{-}}\left(m x_{-}-\right.$gate $)$

Here gate is any of the above four variables, $t$ is time, $t_{-}$is the time constant of the particular type of channel, and $m x_{-}$ is the maximum conductance of this type of channel. $t_{-}$and $m x_{-}$are also defined by equations:

$$
\begin{aligned}
t_{-} & =\frac{1}{\alpha_{-}+\beta_{-}} \\
m x_{-} & =\frac{\alpha_{-}}{\alpha_{-}+\beta_{-}}
\end{aligned}
$$

where

$$
\begin{aligned}
& \alpha_{-}=\frac{A 1+B 1 V}{C 1+\exp \left(\frac{D 1+V}{E 1}\right)} \\
& \beta_{-}=\frac{A 2+B 2 V}{C 2+\exp \left(\frac{D 2+V}{E 2}\right)}
\end{aligned}
$$


Here $V$ is again the membrane potential; $A i, B i, C i, D i$ and $E i$ are parameters to be set for each type of neuron and channel.

\subsubsection{Synapses}

The time evolution of each type of synapse is implemented as the subtraction of 2 exponential functions, the opening function $o$ and the closing function $c$. These functions are increased stepwise: every time a presynaptic spike is fired (zero crossing), the synaptic functions are increased with the fixed value of 10 . Then the functions decay exponentially.

In Eq. (1), $I_{s y n}$ stands for the synaptic input. This is, for neurons $i$ and $j$, defined as:

$I_{s y n}(i, j)=\operatorname{Gmx}(c-o)\left(E r-V_{j}\right) C S(i, j)$

where $G m x$ and $E r$ are the maximum conductance and the reversal potential of that type of synapse respectively, $V_{j}$ is the membrane potential of post-synaptic neuron, $o$ and $c$ are the opening and closing functions of the synapse, and $C S(i, j)$ is the connection strength of this particular synapse. The decay of the opening and closing function is described by the equations

$$
\begin{aligned}
& \frac{d c}{d t}=-\frac{1}{\tau_{c}} c \\
& \frac{d o}{d t}=-\frac{1}{\tau_{o}} o
\end{aligned}
$$

where the $\tau_{o}$ and $\tau_{c}$ are parameters for each type of synapse.

\section{Results}

\subsection{Single neuron models}

Our first objective was to build models of each of the known spinal neuron types (Roberts, 2000). Spinal and caudal hindbrain neurons that are active during swimming have been classified into seven types. The sensory initiation pathway consists of sensory RB neurons that innervate the skin and excite two types of sensory pathway interneuron: dlc (dorsolateral commissural) INs cross to excite CPG neurons on the opposite side and dla (dorso-lateral ascending) INs excite CPG neurons on the same side. The CPG consists of excitatory interneurons (dINs or descending INs; Li et al., 2006; note that we have not separated these neurons into 2 classes as was done by $\mathrm{Li}$ et al.), interneurons producing recurrent inhibition (aINs or ascending INs) and reciprocal inhibition (cINs or commissural INs), and finally motoneurons (MNs).

We have divided these neurons into four groups based on their very clearly different and characteristic firing responses to injected current. We then set parameter values in order to match each model to the physiological properties of each individual neuron type. In life, the properties of individual neurons within each type vary and without using population models we cannot model this variability. Our aim was to produce model neurons whose properties encapsulated the main physiological features of each neuron type. This would allow us to explore the significance of the different properties in the behaviour of the swimming circuit. Unless stated otherwise, we used the generic equations above, and parameters based on Dale (1995b), but for simplicity we have not used any calcium currents. All neurons have fast and slow $\mathrm{K}$ channels. Where Dale used two discontinuous functions to represent the potassium currents, we use two continuous functions instead.

For all types of neurons, the measured parameters are listed in Table 1 and the model neuron parameters are listed in Tables 2 and 3. Input resistance and resting potential were matched by setting the leak conductance and leak reversal potential. To set the firing threshold, the D-parameters of all currents, and some of the A-parameters, needed to be changed from those of Dale. One method to adjust the firing threshold (Roberts and Tunstall, 1990) involves decreasing the spiking threshold by an amount $X$ for all currents, as follows:

$$
\begin{aligned}
& A^{\prime}=A+X * B \\
& D^{\prime}=D+X
\end{aligned}
$$

Then $A^{\prime}$ and $D^{\prime}$ are the new values. In several model neurons, we had to modify the maximum conductances of the ionchannels to match recorded responses.

A detailed explanation of how all parameter settings were obtained, is given in the Appendix. Here we restrict ourselves to a short description of the neurons and example outputs from model and physiology.

\subsubsection{Repetitively firing neurons}

aINs and MNs are most like Dale's original modelled neurons (1995b). They fire repetitively to sustained injected current (Fig. 1), but there are differences, for example in input resistance and firing frequency during current injection ( $\mathrm{Li}$ et al., 2002).

\subsubsection{Single-spiking neurons}

dINs and RBs fire a single, long-duration action potential at the start of current injections and only rarely give any further response (Fig. 2; Li et al., 2003, 2006). A unique property of dINs is that they never fire repetitively to sustained current, no matter how strong it is. 
Table 2 Parameters for all modelled neurons: capacitance (in $\mathrm{pF}$ ), the conductances (in $\mathrm{nS}$ ) and reversal potentials (in $\mathrm{mV}$ ) of their currents

\begin{tabular}{|c|c|c|c|c|c|c|c|}
\hline & aIN & $\mathrm{MN}$ & $\mathrm{dIN}$ & $\mathrm{RB}$ & dlc & dla & cIN \\
\hline $\mathrm{C}$ & 4.00 & 4.00 & 4.00 & 4.00 & 4.00 & 4.00 & 4.00 \\
\hline g_Leak & 1.3514 & 2.4691 & 3.6765 & 4.3573 & 2.3364 & 0.6964 & 4.8544 \\
\hline V_Leak & -54.00 & -61.00 & -51.00 & -70.00 & -66.00 & -63.00 & -60.00 \\
\hline g_Na & 150.00 & 110.00 & 210.00 & 120.00 & 420.00 & 150.00 & 500.00 \\
\hline V_Na & 50.00 & 50.00 & 50.00 & 50.00 & 50.00 & 50.00 & 50.00 \\
\hline g_Kfast & 15.00 & 8.00 & 0.50 & 1.50 & 70.00 & 70.00 & 30.00 \\
\hline V_Kfast & -80.00 & -80.00 & -80.00 & -80.00 & -80.00 & -80.00 & -80.00 \\
\hline g_Kslow & 2.50 & 1.00 & 3.00 & 8.00 & 10.00 & 5.00 & 20.00 \\
\hline V_Kslow & -80.00 & -80.00 & -80.00 & -80.00 & -80.00 & -80.00 & -80.00 \\
\hline g_A & & & & & & & 30.00 \\
\hline V_A & & & & & & & -80.00 \\
\hline
\end{tabular}

Table 3 Detailed parameters for the currents in the modelled neurons

\begin{tabular}{|c|c|c|c|c|c|}
\hline & A & B & $\mathrm{C}$ & $\mathrm{D}$ & $\mathrm{E}$ \\
\hline \multicolumn{6}{|l|}{ aIN/MN } \\
\hline alpha $\mathrm{m} \_\mathrm{Na}$ & $8.67 / 13.26(8.67)$ & $0(0)$ & $0.5(1)$ & $-13.01 /-5.01(-1.01)$ & $-18.56 /-12.56(-12.56)$ \\
\hline beta $\mathrm{m} \_\mathrm{Na}$ & $5.73(3.82)$ & $0(0)$ & $1(1)$ & $-2.99 / 5.01(9.01)$ & $9.69(9.69)$ \\
\hline alpha h_Na & $0.04(0.08)$ & $0(0)$ & $0(0)$ & $15.8 / 28.8(38.88)$ & $26(26)$ \\
\hline beta h_Na & $4.08 / 2.04(4.08)$ & $0(0)$ & $0.001(1)$ & $-19.09 /-9.09(-5.09)$ & $-10.21(-10.21)$ \\
\hline alpha N_Kfast & $3.1(3.1)$ & $0(0)$ & $1(1)$ & $-35.5 /-27.5(-29.5)$ & $-9.3(-23.3)$ \\
\hline beta N_Kfast & $1.1 / 0.44(0.44)$ & $0(0)$ & $1(1)$ & $0.98 / 8.98(6.98)$ & $16.19(16.19)$ \\
\hline alpha N_Kslow & $0.2(0.16)$ & $0(0)$ & $1(1)$ & $-10.96 /-2.96(-4.69)$ & $-7.74(-7.74)$ \\
\hline beta N_Kslow & $0.05(0.04)$ & $0(0)$ & $1(1)$ & $-22.07 /-14.07(-16.07)$ & $6.1(6.1)$ \\
\hline \multicolumn{6}{|l|}{$\mathrm{dIN} / \mathrm{RB}$} \\
\hline alpha $\mathrm{m} \_\mathrm{Na}$ & 13.01 & 0 & $4 / 1$ & $-1.01 /-4.01$ & -12.56 \\
\hline beta $\mathrm{m} \_\mathrm{Na}$ & 5.73 & 0 & 1 & $9.01 / 6.01$ & 9.69 \\
\hline alpha h_Na & $0.06 / 0.04$ & 0 & 0 & $30.88 / 29.88$ & 26 \\
\hline beta $\mathrm{h} \_\mathrm{Na}$ & $3.06 / 2.04$ & 0 & 1 & $-7.09 /-8.09$ & -10.21 \\
\hline alpha N_Kfast & 3.1 & 0 & 1 & $-31.5 /-32.5$ & -9.3 \\
\hline beta N_Kfast & 0.44 & 0 & 1 & $4.98 / 3.98$ & 16.19 \\
\hline alpha N_Kslow & 0.2 & 0 & 1 & $-6.96 /-7.96$ & -7.74 \\
\hline beta N_Kslow & 0.05 & 0 & 2 & $-18.07 /-19.07$ & 6.1 \\
\hline \multicolumn{6}{|l|}{ dlc/dla } \\
\hline alpha $\mathrm{m} \_\mathrm{Na}$ & 13.26 & 0 & $3 / 1.2$ & $-3.01 /-9.01$ & -12.56 \\
\hline beta $\mathrm{m} \_\mathrm{Na}$ & 5.73 & 0 & 1 & $6.01 / 1.01$ & 9.69 \\
\hline alpha h_Na & $0.06 / 0.04$ & 0 & 0 & $19.88 / 14.88$ & 26 \\
\hline beta $\mathrm{h} \_\mathrm{Na}$ & $4.08 / 2.04$ & 0 & 0.001 & $-8.09 /-13.09$ & -10.21 \\
\hline alpha N_K & 3.1 & 0 & 1 & $-32.5 /-37.5$ & -9.3 \\
\hline beta N_Kfast & 1.1 & 0 & $2 / 0.6$ & $3.98 /-1.02$ & 16.19 \\
\hline alpha N_Kslow & 4 & 0 & 1 & $-53 /-58$ & -7.74 \\
\hline beta N_Kslow & 0.01 & 0 & 1 & $47 / 42$ & 6.1 \\
\hline \multicolumn{6}{|l|}{$\mathrm{cIN}$} \\
\hline alpha $\mathrm{m} \_\mathrm{Na}$ & 13.26 & 0 & 0.1 & -10.01 & -12.56 \\
\hline beta $\mathrm{m} \_\mathrm{Na}$ & 5.73 & 0 & 1 & 0.01 & 9.69 \\
\hline alpha h_Na & 0.06 & 0 & 0 & 23.8 & 26 \\
\hline beta $h \_\mathrm{Na}$ & 3.06 & 0 & 0.001 & -14.09 & -10.21 \\
\hline alpha N_Kfast & 3.1 & 0 & 1 & -32.5 & -9.3 \\
\hline beta N_Kfast & 1.1 & 0 & 1 & 3.98 & 16.19 \\
\hline alpha N_Kslow & 0.2 & 0 & 1 & -7.96 & -7.74 \\
\hline beta N_Kslow & 0.05 & 0 & 0.5 & -19.07 & 6.1 \\
\hline alpha $\mathrm{m} \_\mathrm{A}$ & 12.025 & 0 & 0.5 & -10.01 & -12.56 \\
\hline beta $\mathrm{m} \_\mathrm{A}$ & 14.325 & 0 & 1 & -8.01 & 9.69 \\
\hline alpha h_A & 0.0001 & 0 & 1 & 15.88 & 26 \\
\hline beta h_A & 10 & 0 & 500 & -22.09 & -10.21 \\
\hline
\end{tabular}

Note: The original parameters from Dale (1995b) are added between brackets in the top table (with aIN parameters). 

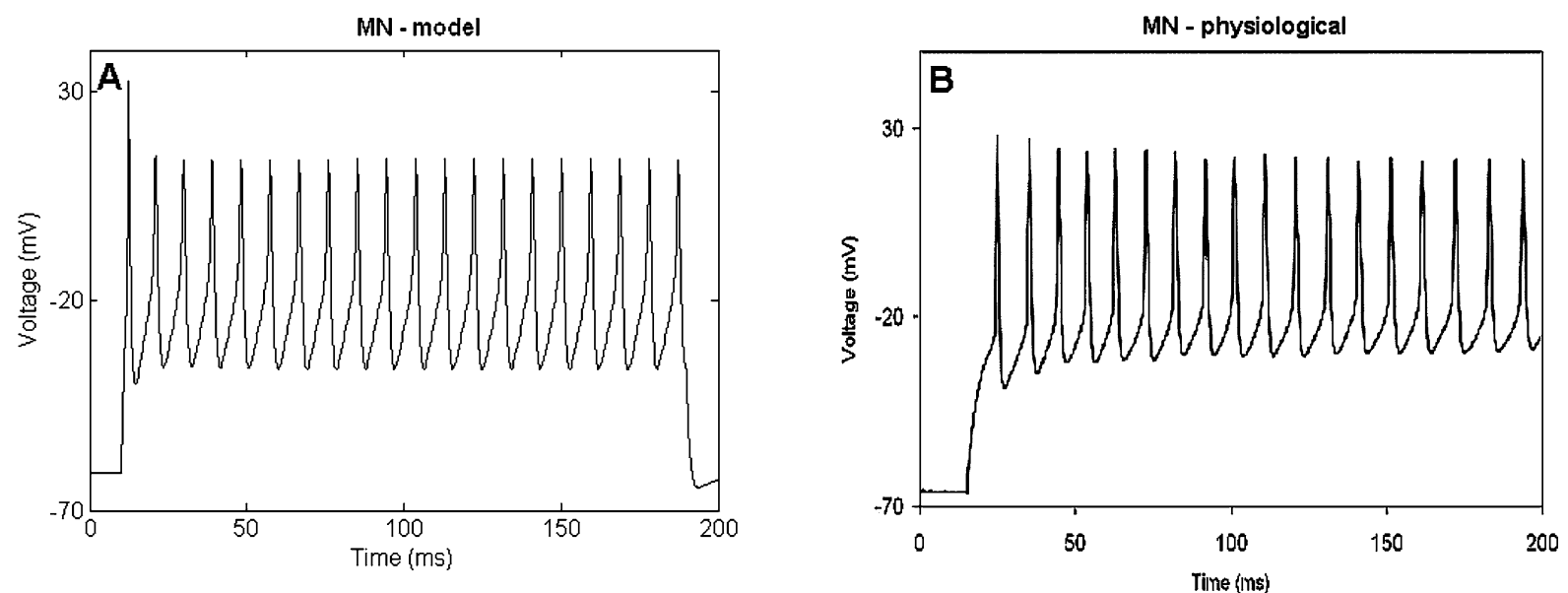

Fig. 1 Responses of repetitively firing neuron model. (A) Output of model MN firing in response to injected current. (B) Physiological measurement of repetitively firing neuron $(\mathrm{MN})$
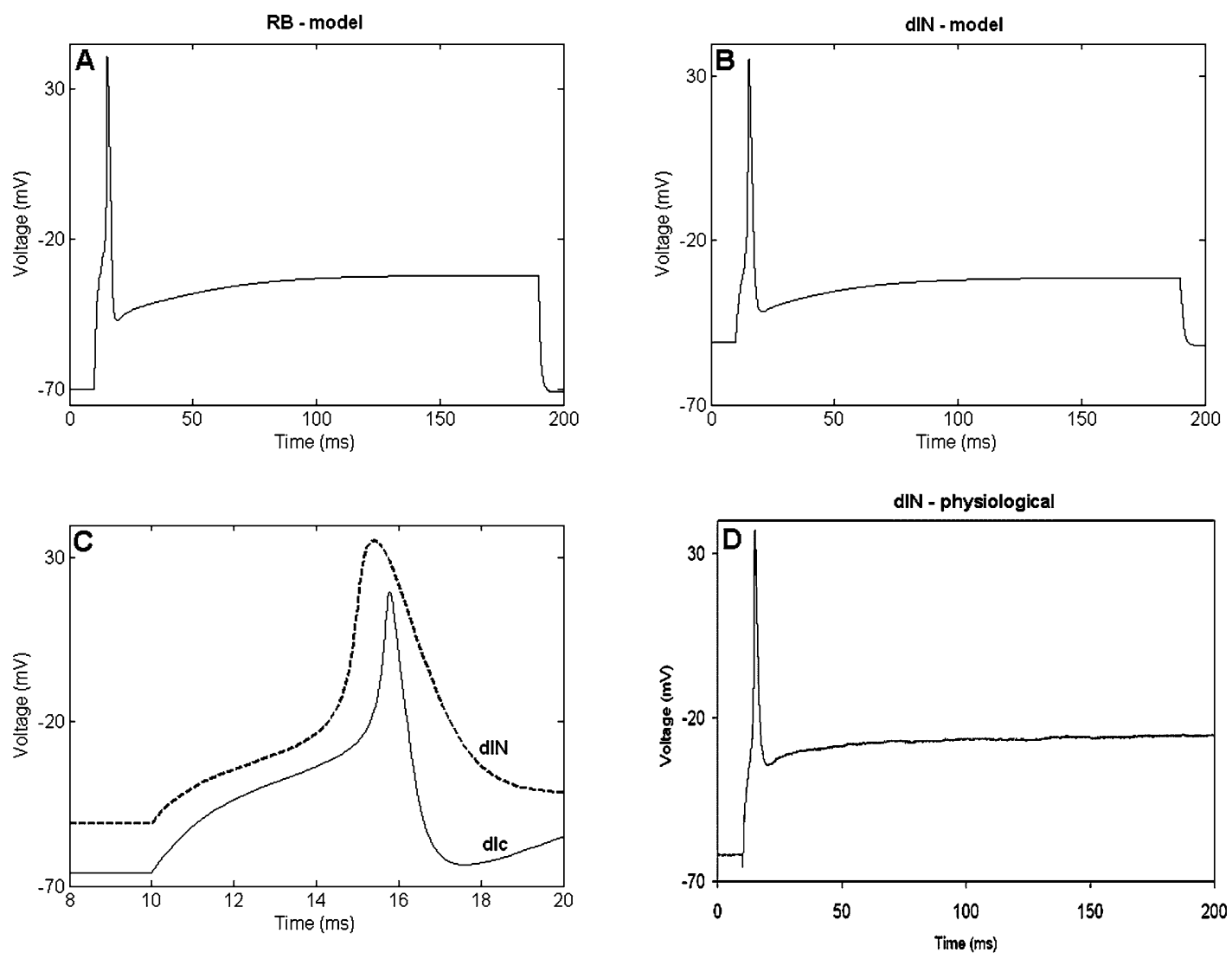

Fig. 2 Responses of single-spiking neuron models to injected current. (A) RB. (B) dIN. (C) Wide dIN spike (thick dashed line) superimposed on thin dlc spike (thin solid line). (D) Physiological measurement from single-spiking neuron (dIN)

A property of dINs, which turns out to be crucial in network behaviour, is that they do not fire on rebound when negative current pulses are given at rest but have the ability to fire on rebound if a negative current pulse of sufficient amplitude is given during depolarisation (see Fig. 4(E) in $\mathrm{Li}$ et al., 2006). This property is also present in our dIN model (Fig. 3). It is not known if this property is exclusive to dINs.

\subsubsection{Repetitively firing neurons with adaptation}

When current is injected into sensory pathway neurons (dlcs and dlas), they fire repetitively, but the frequency drops and firing stops quickly (Fig. 4); (Sillar and Roberts, 1990; Li et al., 2004b). To model this, the slow K-current was modified. 

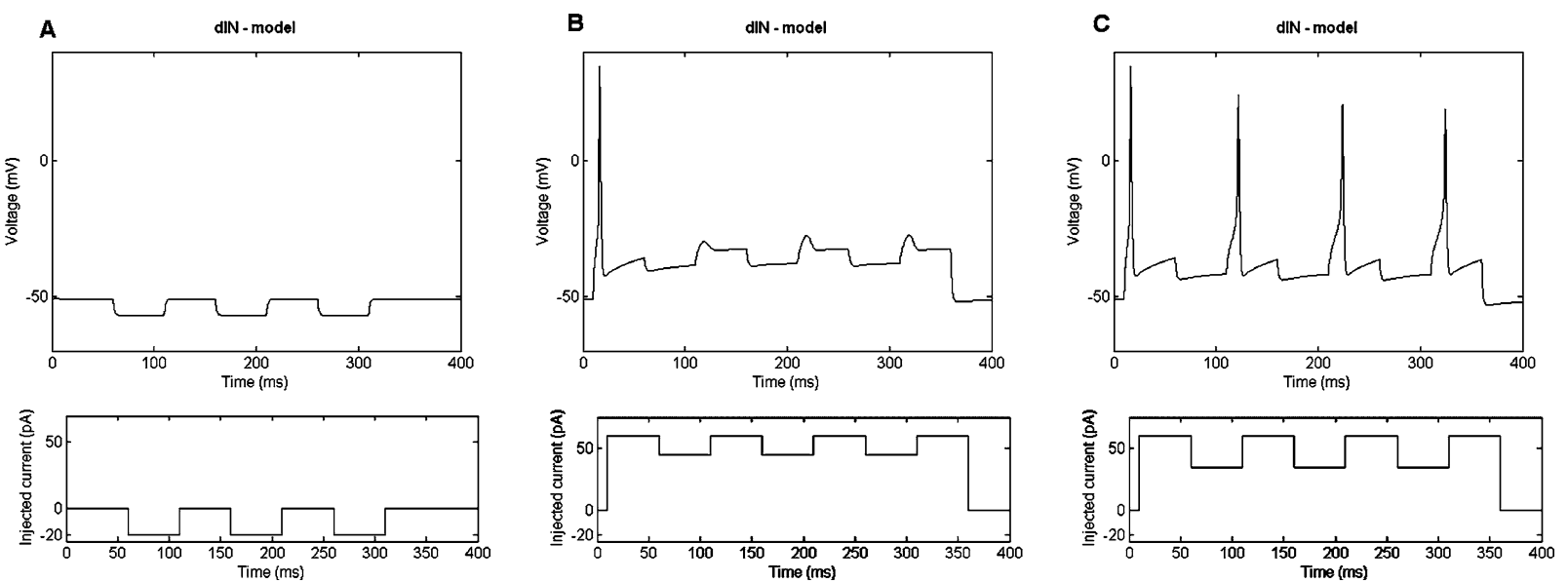

Fig. 3 Rebound firing in dINs. (A) dINs do not fire rebound spikes when negative current is given at resting potential. (B, C) They do fire on rebound after a negative current pulse during depolarisation, if the pulse is of sufficient amplitude
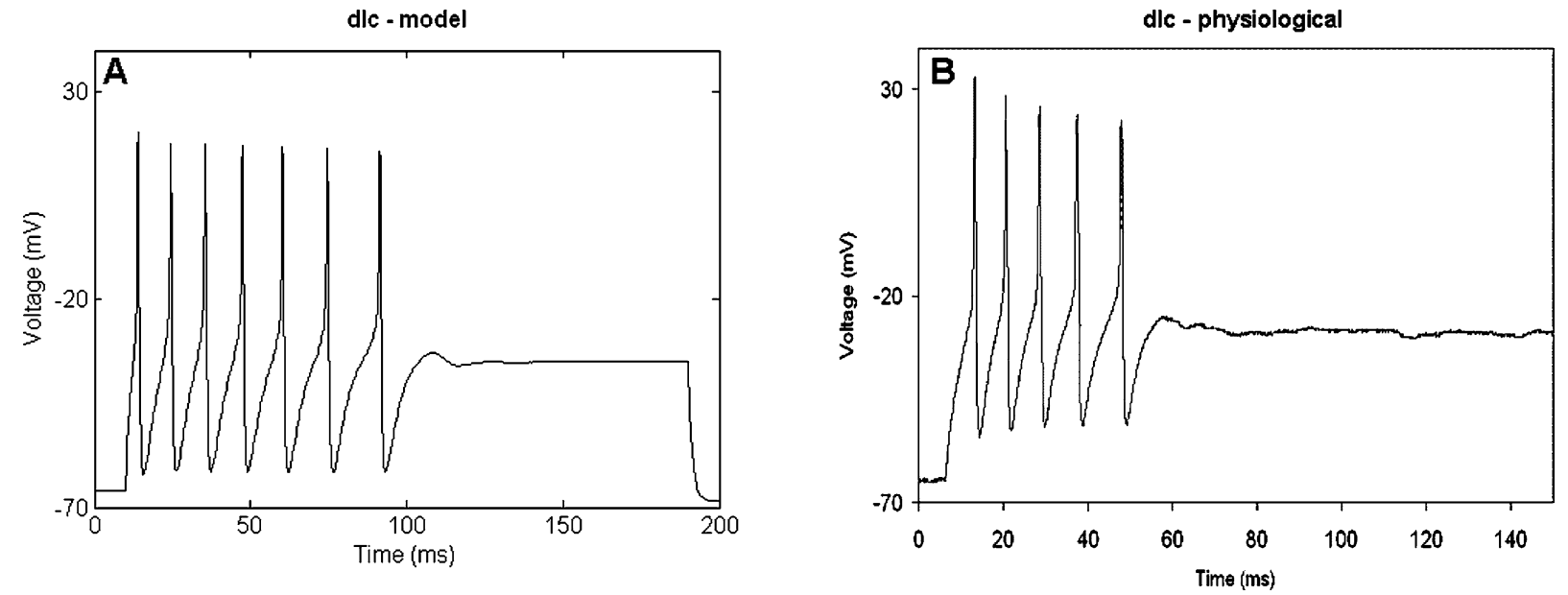

Fig. 4 Responses of repetitively firing neuron model with adaptation. (A) Output of model dlc firing in response to injected current. (B) Physiological measurement of repetitively firing neuron with adaptation (dlc)

\subsubsection{Delayed bursting neurons}

Neurons of one type (cINs) exhibit a delayed burst in response to constant input current (Aiken et al., 2003); see Fig. 4(B) in Li et al. (2004c). Typically, this burst follows a delay which is preceded by a single, initial spike (Fig. 5). To model this property, a further K-current was used, that has both an activation and an inactivation component (Gerstner and Kistler, 2002). A current of this type has previously been described for Xenopus neurons developing in culture (Ribera and Spitzer, 1990).

This current results in an extra term added to Eq. (2).

$$
\begin{aligned}
\text { Currents }= & g_{\text {leak }}\left(V-V_{\text {leak }}\right)+g_{N a} h_{N a} m_{N a}^{3}\left(V-V_{N a}\right) \\
& +g_{\text {Kfast }} n_{\text {Kfast }}\left(V-V_{\text {Kfast }}\right)+g_{\text {Kslow }} n_{\text {Kslow }} \\
& \times\left(V-V_{\text {Kslow }}\right)+g_{A} h_{A} m_{A}^{3}\left(V-V_{A}\right)
\end{aligned}
$$

\subsection{Synapse models}

In our network, we use 3 different types of chemical synapses: glutamatergic AMPAR, and NMDAR mediated excitatory synapses, and glycinergic inhibitory synapses. The NMDAR mediated excitation is voltage dependent (Soffe and Roberts, 1989; Roberts and Tunstall, 1990; Dale, 1995b). Although dINs are known to corelease acetylcholine and glutamate we have not included a separate acetylcholine component. The time course of the acetylcholine current is similar to the AMPA component so we have combined the two as a single "fast" component ( $\mathrm{Li}$ et al., 2004a).

\subsubsection{Synapse parameters}

Table 4 lists the parameters used in the synaptic equations described in Section 2.2.2. Some modifications in the values for $\tau_{o}$ and $\tau_{c}$ were made to fit the experimental data, with the results shown in Figs. 6 and 7. All inhibitory synapses are 
Table 4 Parameters used for chemical synapses

The parameters were based on the references listed, but some were modified slightly to fit the experimental data. The unchanged parameters are in italics.

\begin{tabular}{lllll}
\hline & Inhibitory & AMPA & NMDA & References \\
\hline$G m x(\mathrm{nS})$ & 0.435 & 0.593 & 0.29 & $\begin{array}{l}\text { Galarreta and Hestrin (1997), Gotz et al. } \\
(1997), \text { Chapman et al. (2003) }\end{array}$ \\
$\tau_{o}(\mathrm{~ms})$ & 1.5 & 0.2 & 5 & $\begin{array}{l}\text { Dale }(1995 \mathrm{~b}) \\
\text { Dale }(1995 \mathrm{~b})\end{array}$ \\
$\tau_{c}(\mathrm{~ms})$ & 4 & 3 & 80 & $\begin{array}{l}\text { Roberts and Tunstall (1990), unpublished } \\
\text { measurements }\end{array}$ \\
\hline
\end{tabular}
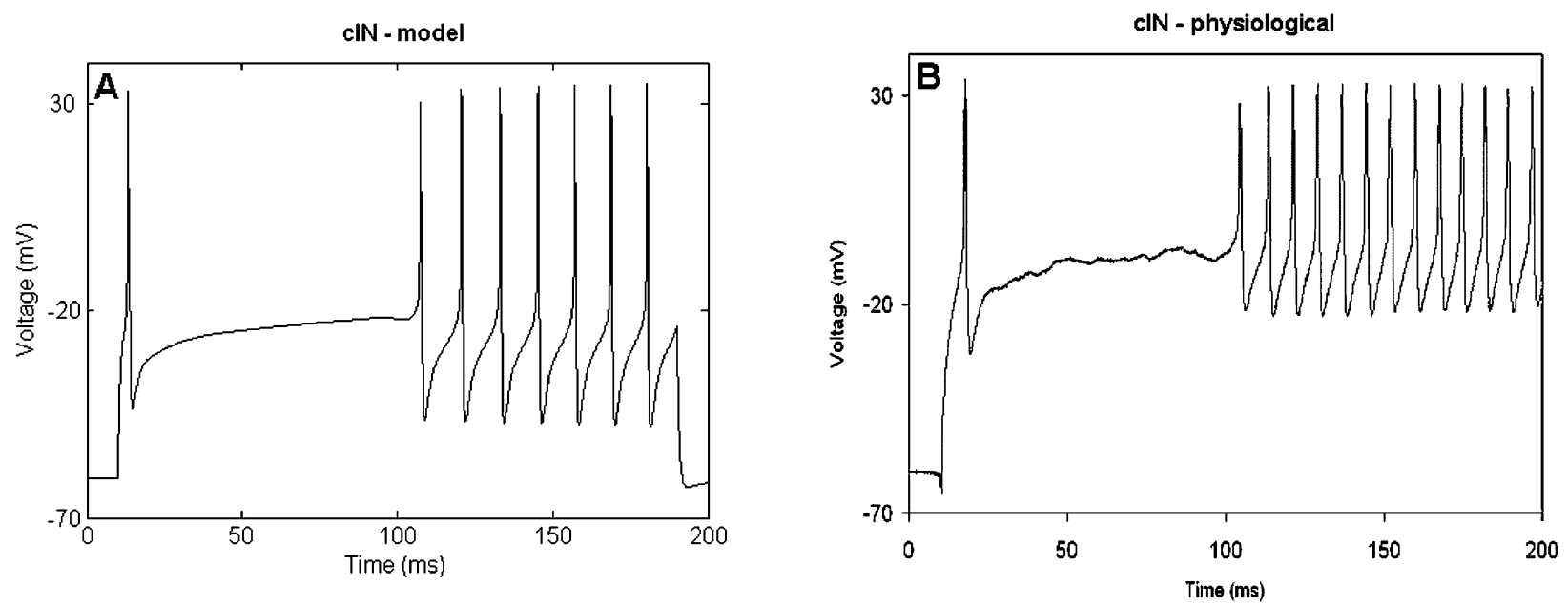

Fig. 5 Responses of delayed firing neuron model. (A) Output of model cIN firing in response to injected current. (B) Physiological measurement of delayed firing neuron (cIN)
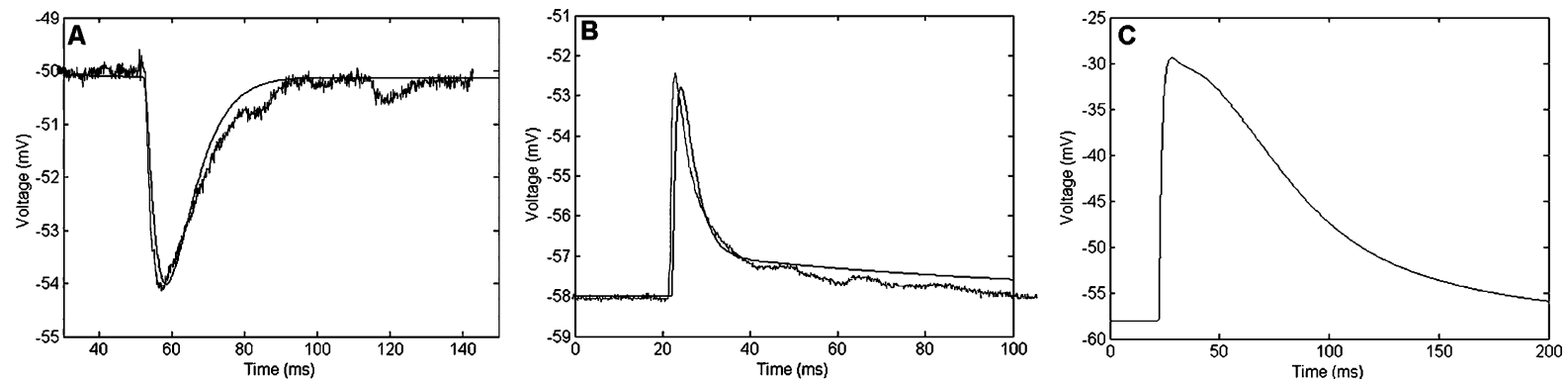

Fig. 6 Post-synaptic potentials at synapses. Lighter straight line $=$ response of a leak-only neuron model (no ionic currents involved), Darker jittery line $=$ physiological measurements. (A) Inhibitory postsynaptic potential. (B) Fast excitatory post-synaptic potential with $50 \%$

the same but in excitatory synapses the ratios of AMPA to NMDA conductances varied (Fig. 6(B) and (C); see Section 3.4.2).

\subsubsection{NMDA voltage dependence}

Voltage dependence of the NMDA synapses is mediated by $\mathrm{Mg}^{2+}$ ions outside the neuron which at resting membrane potential block the NMDA receptor channels, until the postsynaptic neuron is sufficiently depolarized to eject the $\mathrm{Mg}^{2+}$ ions (Nowak et al., 1984). The voltage-dependent NMDA synaptic current is defined by:
AMPA and 50\% NMDA. This ratio is comparable to e.g. the RB to dlc synapse. (C) Slow excitatory post-synaptic potential with 35\% AMPA and $65 \%$ NMDA. This ratio is comparable to the dIN feedback synapse

$I_{s y n}(i, j)=G m x(c-o)\left(E r-V_{j}\right) C S(i, j) X$

$X$ is the voltage dependence factor, defined as:

$$
X=\left(1+0.1\{M g\} \exp \left(-0.08 V_{j}\right)\right)^{-1}
$$

This implementation is based on Lisman et al. (1998). $\{M g\}$ is the extracellular $\mathrm{Mg}^{2+}$ concentration, which is approximated by the constant $0.5 \mathrm{mM}$. The voltage dependency of the NMDA current is shown in Fig. 7 (c.f. Soffe and Roberts, 1989). 


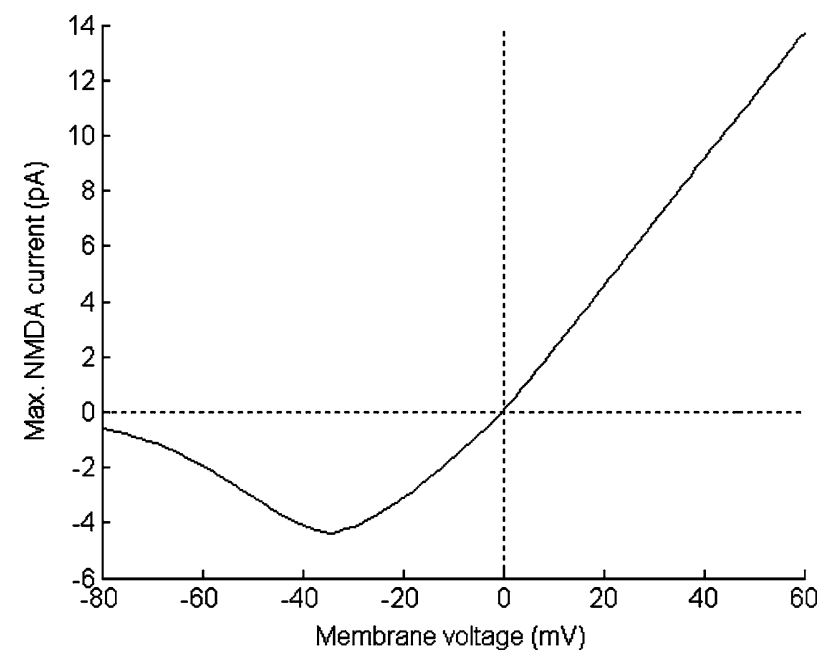

Fig. 7 NMDA voltage dependence. The size of the peak NMDA synaptic current plotted against the membrane potential of a postsynaptic cell modelled with only leak conductances. This plot shows the progressive reduction in synaptic current below $\sim-35 \mathrm{mV}$ reflecting the "negative slope conductance" produced by widely reported $\mathrm{Mg}^{2+}$ block of the NMDA channel

\section{$3.3 \mathrm{dIN}$ rebound properties in small networks}

Experimental work has given direct evidence that the excitatory dINs play a key role in driving the other neurons that are active during the swimming rhythm (Li et al., 2006). However, since these neurons fire only a single spike when depolarised, it is important to consider how they can be made to fire further spikes before exploring the operation of the whole swimming circuit.

As mentioned above, dINs can fire spikes on rebound following negative current injection, provided they are depolarised (Li et al., 2006), and it has been proposed that this property may have a key role in rhythm generation. Neurons depolarized by synaptic excitation could fire on rebound after reciprocal inhibition from neurons on the opposite side of the spinal cord (Roberts and Tunstall, 1990). We therefore tested if the model dIN would fire on rebound following synaptic inhibition. Consider a dIN receiving a fast excitatory synaptic input, followed by an inhibitory one. If the IPSP occurs near resting potential it will not cause any rebound
A

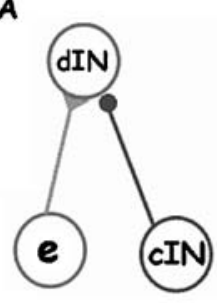

Glutamate

B

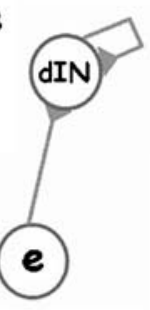

c

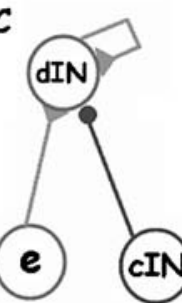

Glycine

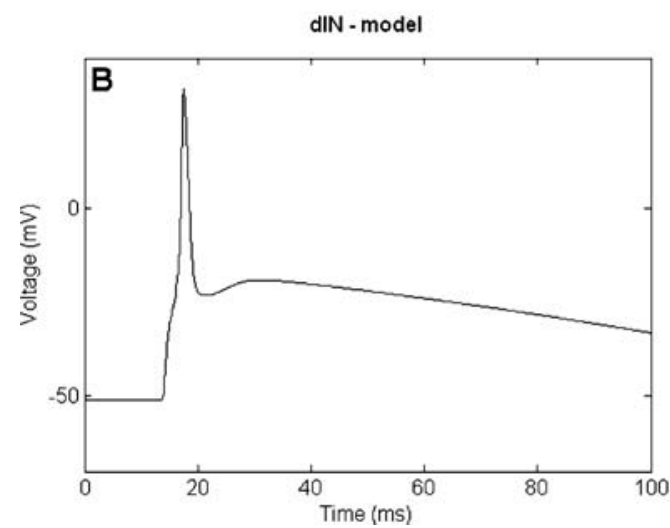

Fig. 8 Post-inhibitory rebound in dINs. Top left: diagrams showing small networks used to create plots A, B and C. (A) dIN response to fast excitatory input (main component is AMPA from neuron e), followed by inhibitory input from a cIN. (B) dIN response to fast excitatory in-
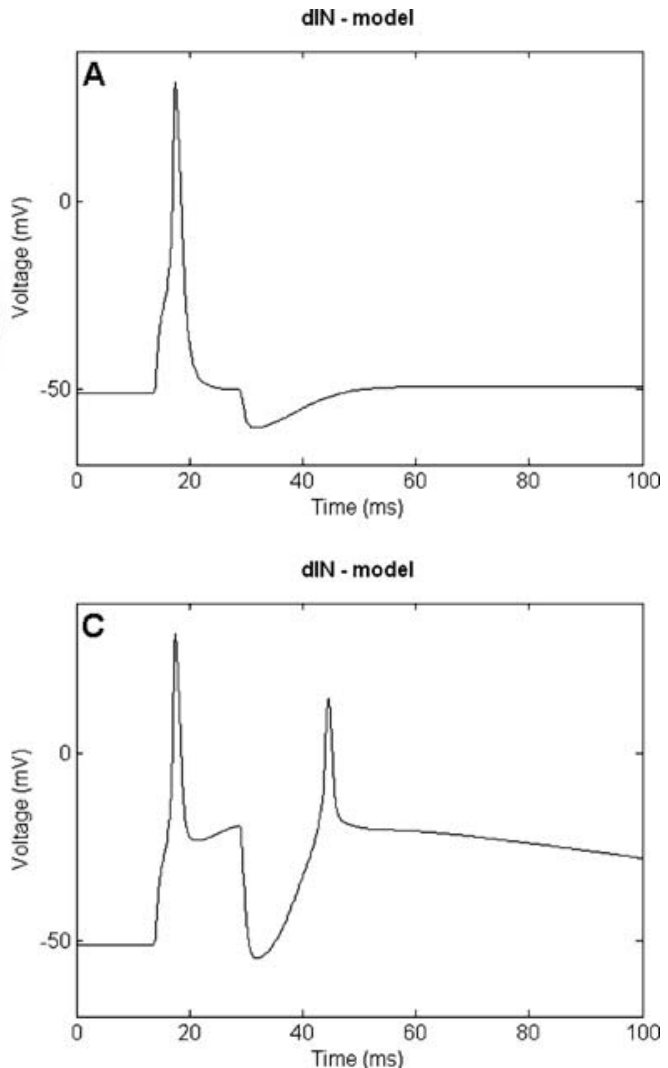

put, followed by slow feedback excitation (main component is NMDA). (C) dIN response to fast excitatory input, followed by slow feedback excitation and then inhibition, causing a rebound spike 
spike (Fig. 8(A)). However, when slow feedback excitation depolarizes the dIN, it will still only fire a single spike after the fast excitatory synaptic input (Fig. 8(B)), but it will fire a rebound spike after the IPSP (Fig. 8(C)).

\subsection{Swimming network}

\subsubsection{Activity of spinal neurons-network diagram}

In our model of the circuitry for swimming and its sensory initiation, single neurons of each type are used to represent what in the real animal are populations of similar neurons. The network architecture is based on the anatomical evidence on axonal projections and the synaptic connections are based on results from paired recordings. The RB neurons are touch sensory neurons innervating the skin. Thus, when the Xenopus skin is touched, the RB neurons fire (Clarke et al., 1984). The RB neurons excite sensory interneurons (dlc and dla), which relay the signals through excitatory synapses to the contralateral and ipsilateral side, respectively ( $\mathrm{Li}$ et al., 2003, b). Here they synapse onto all CPG neurons, including dINs (Li et al., 2003). The dINs in turn excite all CPG neurons on their own side (Li et al., 2006). The other CPG neurons are aINs, which provide ipsilateral, recurrent inhibition ( $\mathrm{Li}$ et al., 2004d), cINs, which provide contralateral, reciprocal inhibition (Dale, 1985; Soffe et al., 2001) and MNs, which connect to the muscles and cause the swimming movements (Soffe and Roberts, 1982).

The model network (Fig. 9) has a left and right side but no length and represents the neurons and connections found in the caudal hindbrain and rostral spinal cord. All ipsilateral synapses were assumed to have a combined axonal-synaptic

\section{Left \\ Right}

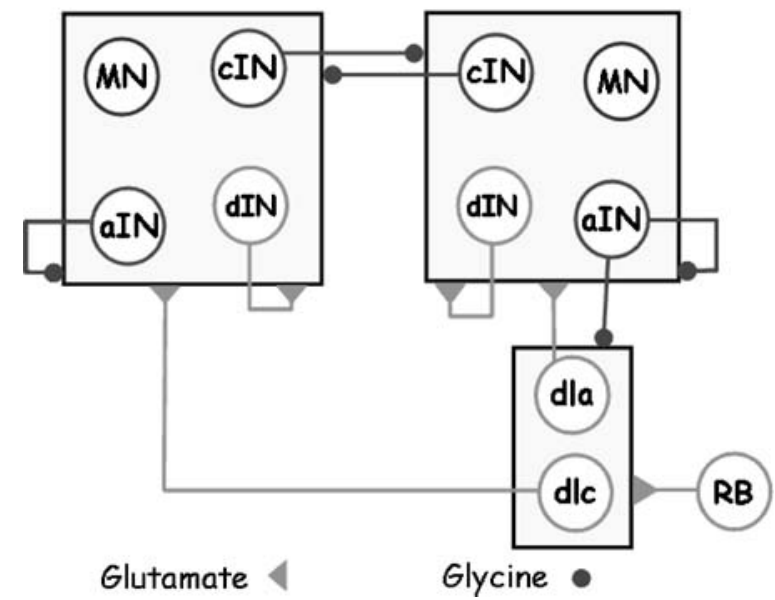

Fig. 9 Diagram of the neuronal network which generates swimming. Larger circles represent each type of neuron, small triangles stand for excitatory synapses and small circles for inhibitory synapses. A synapse to a 'box' means the connection is made to all neurons inside the box delay of $1 \mathrm{~ms}$, all commissural synapses were given a delay of $2 \mathrm{~ms}$ as they are made from axons which have crossed from the other side of the spinal cord.

\subsubsection{Synaptic conductances}

During swimming, some of the maximum conductances were measured directly in physiological experiments (Table 5, on the left). Of those that were not (or could not

Table 5 Physiological maximum conductances (in $\mathrm{nS}$ ) for synaptic connections based on direct voltage clamp measurements and estimates (see notes)

\begin{tabular}{llllllll}
\hline & & \multicolumn{3}{c}{ Measured } & & \multicolumn{2}{c}{ Model } \\
\cline { 5 - 6 } Source & Target & AMPA & NMDA & A:N ratio & & AMPA & NMDA \\
\hline RB & dlc & $5.2^{1}$ & $0.17^{2}$ & $30.0^{3}$ & & 6.77 & 0.16 \\
& dla & $5.2^{1}$ & $0.17^{4}$ & $30.0^{4}$ & & 3.38 & 0.16 \\
dlc & CPG & $1.3^{5}$ & $0.9^{6}$ & $1.5^{6}$ & & 2.92 & 0.96 \\
dla & CPG & $1.3^{7}$ & $0.9^{7}$ & $1.5^{7}$ & & 2.55 & 1.08 \\
dIN & aIN & $1.6^{8}$ & $0.5^{9}$ & $3.3^{\mathrm{A}}$ & & 1.46 & 0.54 \\
& cIN & $3.6^{8}$ & $1.1^{9}$ & $3.3^{\mathrm{A}}$ & & 8.51 & 1.05 \\
& dIN & $4.2^{8}$ & $4.2^{9}$ & $1.0^{\mathrm{A}}$ & & 4.38 & 6.86 \\
& MN & $5.1^{8}$ & $1.5^{9}$ & $3.3^{\mathrm{A}}$ & 3.89 & 1.47 \\
& & Inhibition & & & Model & \\
cIN & aIN & $2.9^{\mathrm{B}}$ & & & 2.86 & \\
& cIN & $2.9^{\mathrm{B}}$ & & & 2.86 & \\
& dIN & $8.1^{\mathrm{B}}$ & & & 9.53 & \\
& MN & $3.9^{\mathrm{B}}$ & & & 3.81 & \\
aIN & cIN & $1.0^{\mathrm{C}}$ & & & 1.11 & \\
& dIN & $2.7^{\mathrm{C}}$ & & & 2.70 & \\
& MN & $1.3^{\mathrm{C}}$ & & & 1.27 & \\
& dlc & $5.0^{\mathrm{B}}$ & & & 6.36 & \\
& dla & $5.0^{\mathrm{B}}$ & & & 6.36 & \\
\hline
\end{tabular}

${ }^{1}$ Calculated from peak current in response to skin stimulation and assuming a reversal potential of $0 \mathrm{mV}$.

${ }^{2}$ Calculated using AMPA conductance and published AMPA/NMDA ratio (Li et al., 2003).

${ }^{3}$ From Li et al. (2003).

${ }^{4}$ Calculated from measured AMPA conductance, assuming the same AMPA/NMDA ratio as for RB-dlc.

${ }^{5}$ Calculated from published peak current (Li et al., 2003) in response to contralateral skin stimulation.

${ }^{6}$ Calculated from measured AMPA conductance and published AMPA/NMDA ratio (Li et al., 2003).

${ }^{7}$ Assumed to be the same as dlc-CPG but unconfirmed.

${ }^{8}$ Peak AMPA currents measured during swimming; conductance calculated assuming a reversal potential of $0 \mathrm{mV}$. For these connections, AMPA is actually total "fast" excitation (AMPA + nACh corelease; Li et al., 2004).

${ }^{9}$ Calculated from measured AMPA conductance and published AMPA/NMDA ratio (Li et al., 2004).

${ }^{\mathrm{A}}$ Recalculated from data used in Li et al. (2004).

${ }^{\mathrm{B}}$ Calculated from maximum peak currents and reversal potentials measured at mid-cycle during swimming.

${ }^{\mathrm{C}}$ Estimated using cIN values and assuming a cIN/aIN ratio of 3. 
A

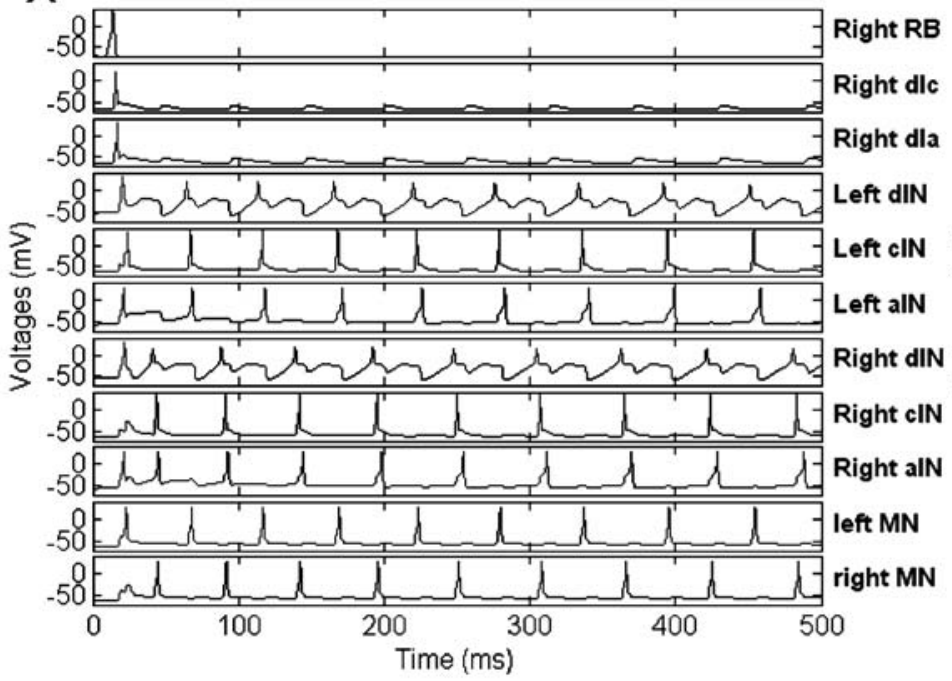

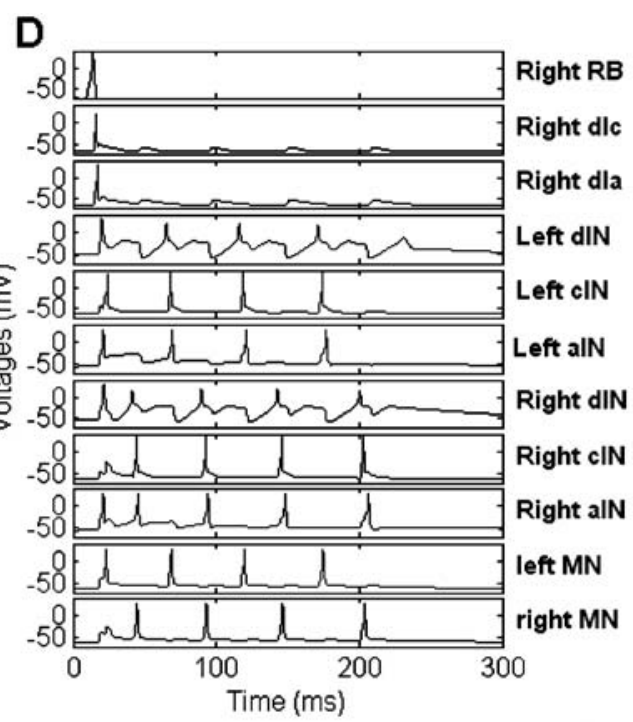

C
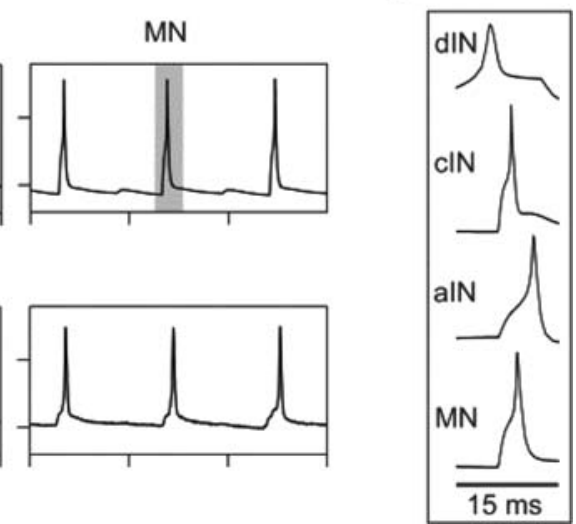

Fig. 10 Swimming activity (A) Voltage traces of all neurons in the network during sustained swimming, produced using the parameter values in Tables 2-5. (B) Comparison of model output (sequences expanded from A) to physiologically recorded swimming activity of different

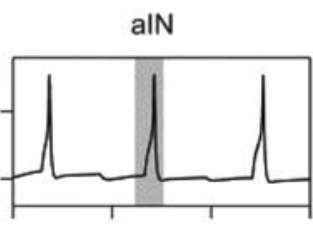

Physiology
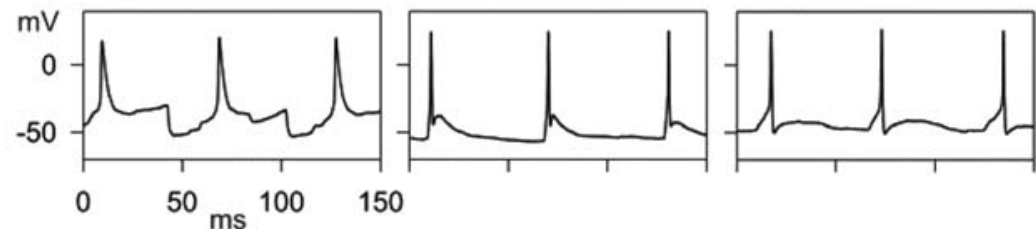

CPG neuron types. (C) dIN firing leads cIN and $\mathrm{MN}$ firing on each swimming cycle and aIN firing is relatively late. Data expanded from B (grey bars). (D) With the strength of dIN feedback excitation reduced, swimming is sustained for only $4-5$ cycles be) directly measured, estimates were made (for example, using AMPA:NMDA ratios measured from unitary synaptic potentials). The synaptic strengths in the model swimming network were set initially to produce conductances whose peak values during swimming (Table 5, on the right) approximate the measured (or estimated) values. These values measured (or estimated) for a particular type of synaptic connection were actually the sum of synchronous inputs from a population of neurons of the same type. Since populations of neurons are represented as individual neurons in the model, each connection type was represented as a single synapse whose conductance, as in a real neuron, was equivalent to that produced by input from a population of neurons.

\subsubsection{Swimming result}

Unlike previous models of the spinal cord, which lacked sensory pathway neurons, a single "stimulus" to one RB neuron (on the right side), leading to a single RB action potential, makes the network enter a swimming state, where left and right sides fire alternating spikes. Exactly as in physiological experiments, the side opposite the stimulus (left) is active first and the firing rate is about $20 \mathrm{~Hz}$ (Fig. 10(A)). As well as producing a clear swimming rhythm, the activity patterns of different neuron types resemble those seen physiologically, in a number of important respects (as shown in Fig. 10(B)). Activity in the dINs is clearly different to that in the other CPG neurons, with a stronger background (tonic) depolarisation 


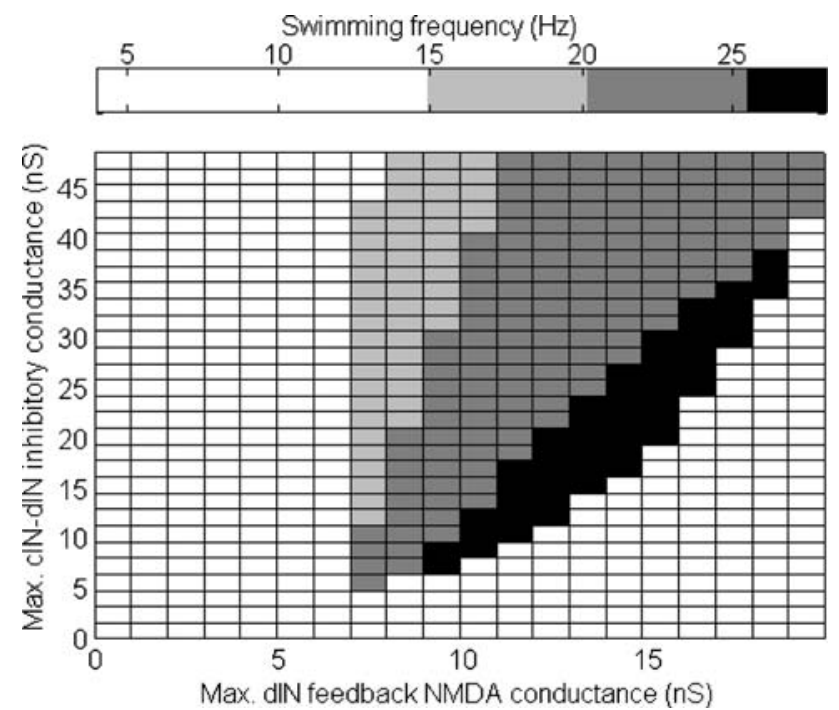

Fig. 11 Surface plot of network swimming frequency, for different maximum conductance values of dIN feedback NMDA excitation and cIN-dIN inhibition. The gray area (both lighter and darker) has reliable swimming at a realistic frequency (15 to $25 \mathrm{~Hz})$

and more prominent reciprocal, mid-cycle IPSPs, followed on each cycle by rebound firing (Li et al., 2006). In contrast: the background depolarisation in the cINs, aINs and MNs is much smaller, and the mid cycle inhibition is hardly visible as the membrane potential is close to the inhibitory reversal potential (Li et al., 2002, 2006). In these neurons the depolarisation is not sufficient for any to fire repetitively (as they are capable of doing to sustained current), or to fire on rebound. Instead, it is the relatively prominent fast excitation from the dINs that drives their single spike on each cycle. As a result, firing in the dINs leads firing in the other neurons

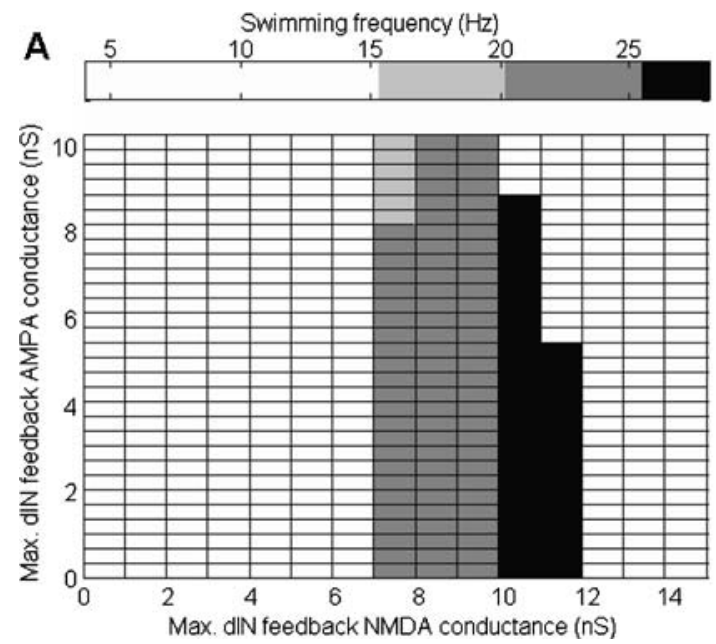

Fig. 12 Influence of dIN feedback NMDA excitation and cIN-dIN inhibition on swimming. (A) Surface plot of swimming frequency, for different maximum conductance values of dIN feedback NMDA and AMPA excitation. (B) Surface plot of swimming frequency, for differ- on the same side (Fig. 10(C)). The relatively weak excitation of aINs means that they fire relatively late on each cycle, as described experimentally ( $\mathrm{Li}$ et al., 2002).

If the level of excitation from dINs was reduced, similar swimming activity was seen but, as in "spinalised" animals where excitation is similarly thought to be reduced (Roberts et al., 1985), activity fails after a few cycles (Fig. 10(D)).

To test the stability of the network, we varied the amplitude of the NMDA-component of the dIN feedback connection and of the cIN-dIN inhibition independently to establish the range over which swimming occurs (Fig. 11). These synapses were chosen because they together control the rebound firing which drives the swimming network. In the gray area (both lighter and darker), there is stable swimming: the MNs give alternating output in the normal frequency range for swimming ( 15 to $25 \mathrm{~Hz}$ ). Broadly: as the strength of dIN feedback excitation is increased from its lowest value, the frequency and number of swimming cycles increases until it becomes self sustaining; increasing the cIN-dIN inhibition decreases the frequency.

The strengths of other synapses can also play important roles in the network, but these are not as crucial to the production of alternating firing as the rebound firing mechanism. For example in Fig. 12(A), a frequency plot is shown for the swimming network, when varying the AMPA and NMDA components of dIN feedback excitation. While variation in the NMDA strength clearly influences swimming frequency, variation of the AMPA strength has much less effect. Figure 12(B), similarly shows that varying cIN-dIN inhibitory conductance results in significant changes in swimming output, whereas varying cIN-cIN inhibition doesn't have any effect on swimming frequency.

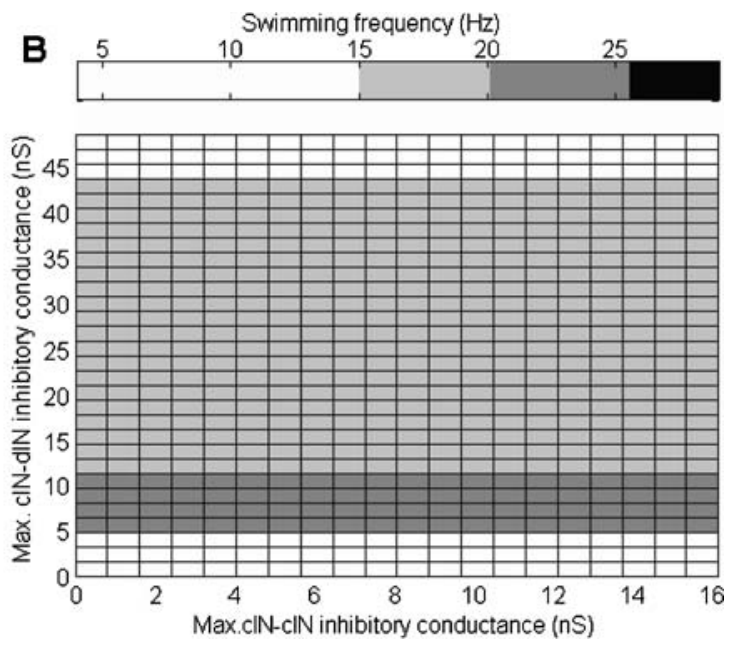

ent maximum conductance values of cIN-cIN and cIN-dIN inhibition. Note: cIN-cIN inhibition has a smaller range, since its physiological conductance strength is much lower than the cIN-dIN inhibition 


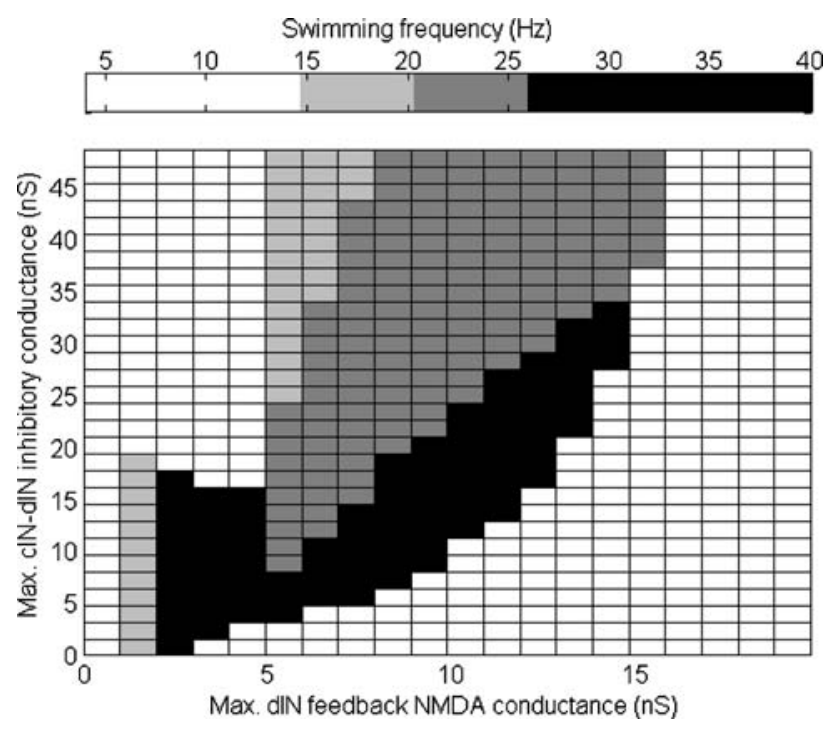

Fig. 13 Frequency plot where dINs have the repetitive firing properties of aINs. The gray area (both lighter and darker) has realistic swimming output ( 15 to $25 \mathrm{~Hz}$ ) and the black area has high frequency output, with synchrony between the two sides

\subsection{Testing the importance of type-specific neuron models}

\subsubsection{Importance of single-spiking property of dINs}

To investigate the importance of type-specific individual neuron models, we have focussed on the dINs. They provide excitation to drive the firing of the other active neurons on the same side, provide feedback excitation within each side, and receive reciprocal inhibition from contralateral cINs. These connections are crucial to allow the dINs to fire on rebound and therefore to drive swimming. Having established the stable area of swimming with dINs in the network (Fig. 11), we changed the firing properties of the dINs.

We first gave them the repetitive firing properties of aINs. This led to unstable activity. The frequency plot (Fig. 13) shows that the area of stable swimming (2 levels of gray) is partly enclosed by an area of high frequency with synchrony between the two sides (black). Comparing this to Fig. 11, shows that replacing the dINs by aINs reduces the area of stable swimming, and causes a large area of synchronous firing on both sides of the body which would not lead to meaningful behaviour (see Fig. 15).

When dINs were given the properties of cINs they were unable to fire on rebound unless unrealistically high conductances were used, and in those cases, only synchrony or unstable firing patterns were produced. Substituting the properties of dINs suggests that their type-specific "singlespiking" properties are important for the generation of stable swimming in this network model.

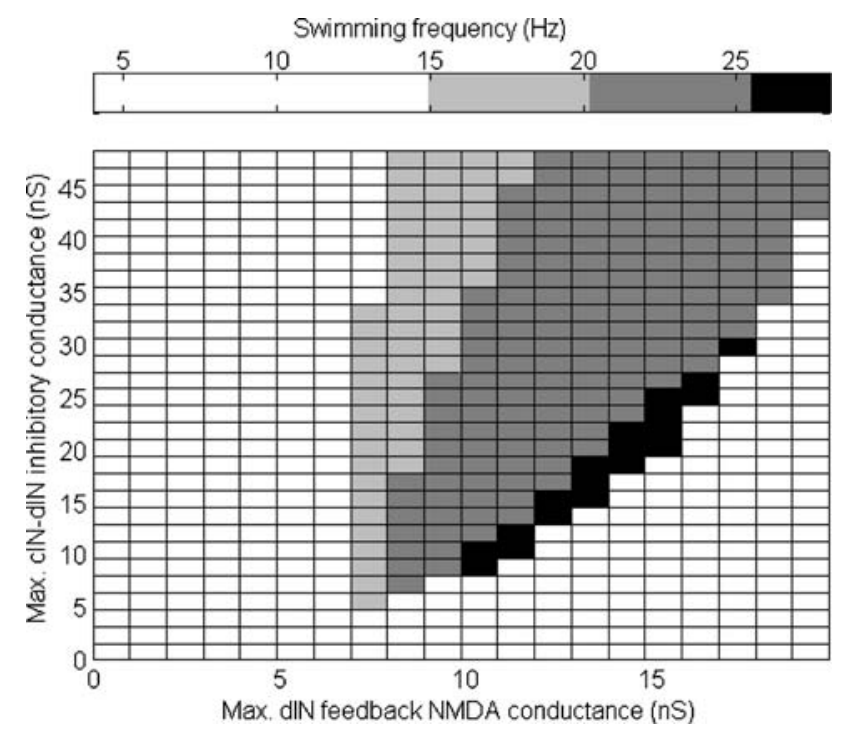

Fig. 14 Surface plot of swimming frequency with all neurons having dIN properties. The gray area (both lighter and darker) has stable swimming at a realistic frequency $(15$ to $25 \mathrm{~Hz})$

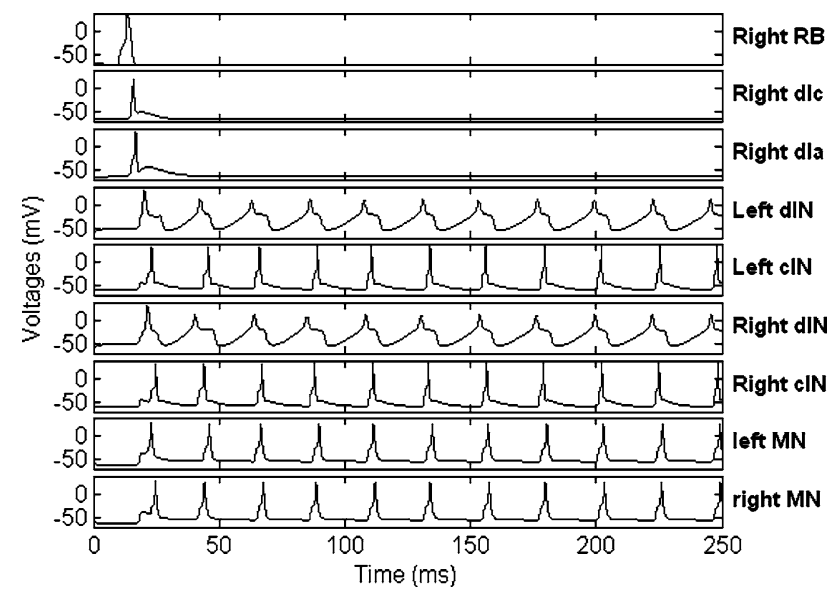

Fig. 15 Example network output when aINs are removed. The network exhibits synchronous firing on both sides of the body, instead of alternating spikes

\subsubsection{Importance of different neuron types}

Since previous model networks have used a single generic neuron model (Roberts and Tunstall, 1990; Dale, 2003; Li et al., 2006), we made a network where all neurons had the same properties. In this case, our generic neuron model had the single-spiking property of dINs, rather than showing repetitive firing (as in Dale, 1995b), since our modelling already suggested this was a key feature. Figure 14 shows a reliability surface for this network. There is swimming activity in about the same parameter region as in the regular swimming network (Fig. 11). This is perhaps not surprising, given that all CPG neurons typically fire only once per cycle during swimming. However, it does raise the question of why 
most of the CPG neurons can fire repetitively (see Discussion).

\subsubsection{Importance of aINs in the swimming network}

The key neurons in the swimming network appear to be the dINs providing excitation and the cINs providing reciprocal inhibition. We know that, in addition, aINs provide recurrent inhibition to CPG neurons (Li et al., 2004d), and this inhibition is particularly prominent as swimming is initiated.

When aINs are removed from the model network by setting their synaptic conductances to zero, the initiation of swimming always fails, leading only to high-frequency synchronous firing of MNs on both sides of the body. An example output is shown in Fig. 15. This is the case even after varying several other synaptic conductances: the dlc/dla-dIN AMPA connection, dIN-dIN NMDA connection and cINdIN inhibitory connection were all varied over wide ranges, without producing good swimming behaviour. We can explain this role of aINs as follows. Following stimulation of the right sensory RB neuron in our model, the right dla and dIN both excite the right aIN which fires quite quickly, and prevents the right cIN and MN from firing. Thus only the left cIN and MN fire, leading to rebound spiking in the right $\mathrm{dIN}$, and swimming is started. When the aINs are removed, this process, that ensures swimming starts on only one side, is lost.

By increasing the dIN-aIN synaptic connection strength significantly (by a factor of 4), one can stop the network swimming: both aINs fire much sooner in the cycle, before the cINs do, and suppress the cIN spike. Thus any reciprocal inhibition is stopped, and the opposite dIN will receive no inhibition from which to fire on rebound. A similar effect is seen when the strength of aIN-CPG inhibition is increased sufficiently.

\subsection{Role of aINs in the sensory pathway}

In addition to their connections to other pattern generator neurons, aINs play a role in controlling sensory pathways. During swimming, sensory input from the skin via RB neurons is inhibited at certain phases of the swim cycle so that it does not lead to responses that are in conflict with swimming (Sillar and Roberts, 1988). We now know that this gating inhibition of dlc and dla interneurons comes from aINs ( $\mathrm{Li}$ et al., 2002). Since these connections are a part of our model network, sensory gating can operate. If the RB neuron gets an extra stimulus during swimming, the timing of the stimulus in the swimming cycle determines whether the dlas and dlcs will fire (Fig. 16). Only if they fire will their excitation reach the CPG and lead to an increase in swimming frequency (Fig. 17).
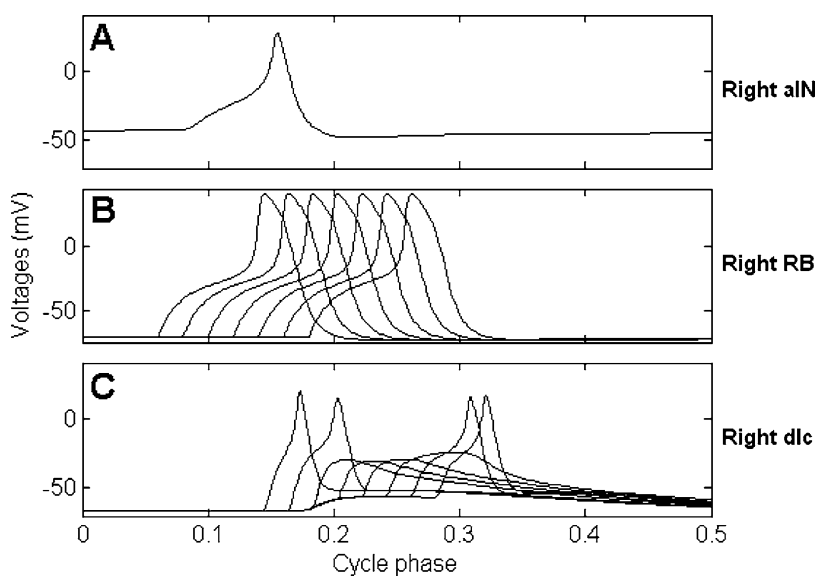

Fig. 16 Gating of sensory transmission from RB to dlc. Experiments done in the complete network model, but only the output of the three most important neurons is shown. (A) aIN spike near the start of a cycle of swimming (phase 0). (B) Series of spikes in RB from separate stimuli given at different times relative to the aIN spike. (C) depending on the relative timing of aIN and RB spikes, dlc firing can be suppressed by the aIN inhibition

\section{Discussion}

In detailed modelling, based on the physiology of neuronal networks, the goal is to match a real-life system as closely as possible, to investigate how it works. Choices always have to be made on the level of detail, and recently it has become clear that even in larger neuronal model-networks, the properties of individual neurons can play an important role (Bem et al., 2005; Jezzini et al., 2004). Our aim was to use neuron and neuronal network modelling to investigate the significance of the type-specific differences in properties of spinal neurons in the swimming network of the young Xenopus tadpole.

We have based our neuron modelling on data from Dale (1995a) who made whole-cell patch recordings from isolated Xenopus spinal neurons and defined the characteristics of their voltage-gated currents. On the basis of this data and the assumption that spinal neurons all had similar properties, Dale built a generic CPG-neuron (Dale, 1995b) which was then used to model different neuron types in the spinal CPG network by giving them different output synapses. His generic neuron model fires repetitively in response to near threshold injected current at frequencies just higher than swimming frequencies. As a result, when these neurons are connected into a reciprocal inhibitory half-centre model of the spinal CPG, it can generate alternating swimming activity in the normal frequency range (Dale, 2003).

More recent, in situ whole-cell recordings show that different spinal neuron types have type-specific properties that include different spike firing properties (Aiken et al., 2003; Li et al., 2002, 2003, 2004b, d). There is broad agreement about the spike firing characteristics of RB sensory neurons, 


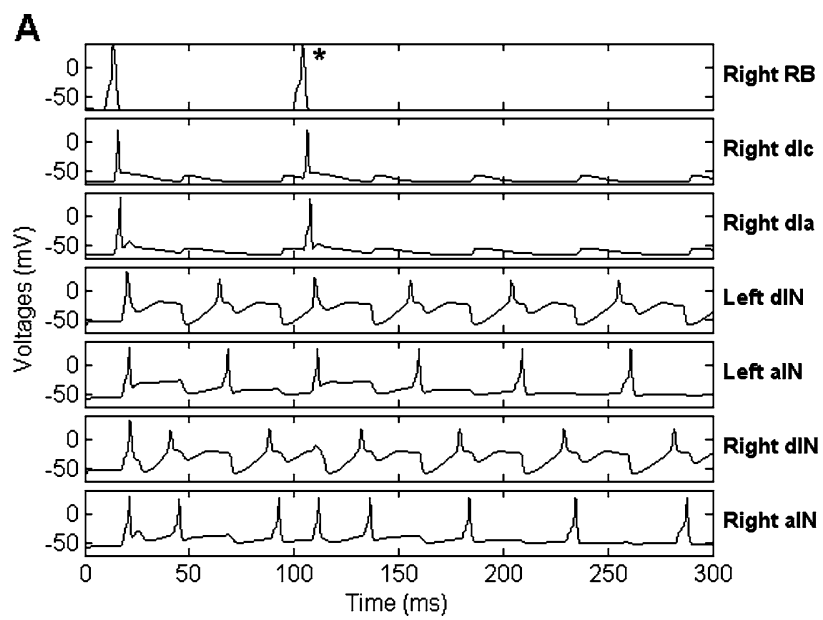

Fig. 17 Effects of sensory gating during swimming. Two examples of swimming runs, in which the RB gets a second stimulus during swimming (*). (A) The second RB spike causes the dlc and dla to fire, influencing the behaviour of the whole network (cINs and MNs

sensory pathway interneurons and reciprocal inhibitory cIN interneurons. However, Aiken et al. (2003) suggest that excitatory CPG dINs fire repetitively on the basis of recordings from 5 neurons identified by activity during swimming and possession of a descending axon. In contrast, our evidence shows that dINs fire a single characteristically long-duration spike to current ( $\mathrm{Li}$ et al., 2006). It is based on 103 paired recordings from dINs with anatomy and the direct physiological demonstration that these neurons excited more caudal spinal CPG neurons.

Using our physiological evidence on the properties of both neurons and their synapses, we have built detailed models for each individual type of neuron in the Xenopus tadpole spinal cord. We have modelled 7 types of neurons, which exhibit 4 very distinct types of firing response to injected current, but which all differ further for example in resting potential, input resistance and spike shape. We have also used realistic models for synaptic connections, incorporating different channel opening and closing rates, and the voltage dependence in NMDA receptor channels. Using these neurons and synapses and their known patterns of connectivity, we have made a point model of the rostral spinal network of the young Xenopus tadpole with left and right sides but no length. When the measured physiological conductances were closely matched, single stimuli to the sensory neuron in this network were able to initiate swimming activity. Despite what may seem fairly radical simplifications, our model neurons generate voltage records that are a remarkably good fit to real recordings from the different types of neuron during swimming (see Fig. 10). Also, we know from lesion studies that a very short region of the CNS can generate prolonged swimming (Li et al., 2006). In such short regions spike activity within each neuron class is nearly synchronous, so

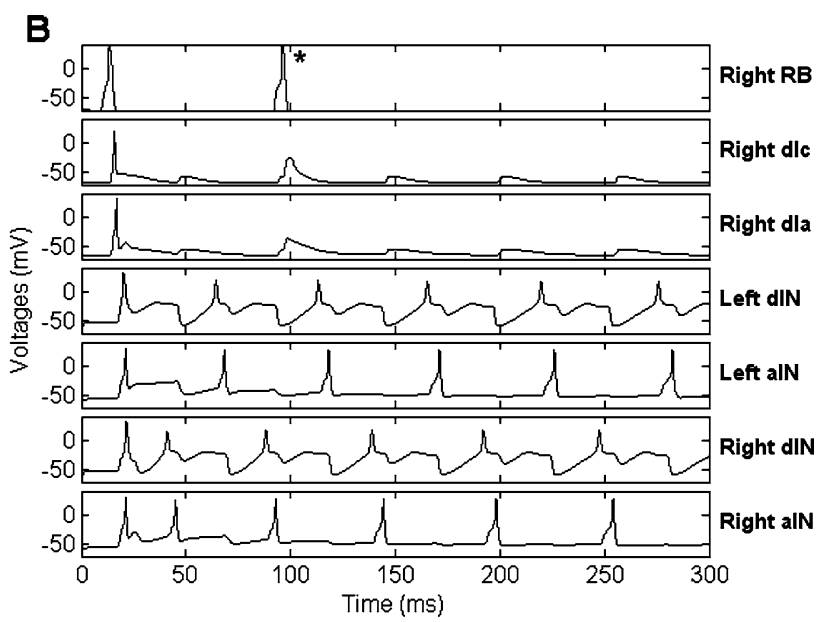

omitted for clarity): the frequency of the following two cycles is increased. (B) The RB spike now coincides with inhibition of the dlc and dla by the ipsilateral aIN. As a result, their firing is suppressed, sensory transmission is gated out and network behaviour is unaffected

representing a small population of 30 or so neurons by a single neuron seems not unreasonable.

The model network has other features that make it much more like the real network than previous models (Dale, 1995b; Tunstall et al., 2002). For example, it incorporates sensory and sensory pathway neurons so it can be activated by a brief "natural" stimulus. This contrasts with earlier models that required delayed stimuli to both sides. As in the spinal tadpole, the first activity in swimming is on the side opposite that stimulated (Zhao et al., 1998). In the model network this results from stronger contralateral synapses (right dlc to left CPG) than ipsilateral (right dla to right CPG; see Fig. 9). Also the aINs play an important role in this process, by firing early and suppressing the firing of the right cINs and MNs. Thus the left side starts firing, inhibiting the right side, which leads to rebound spikes and the process of swimming is started. An important feature that emerges in the model network from the connections of inhibitory aINs onto sensory pathway dlc and dla interneurons is sensory gating (Sillar and Roberts, 1988) where extra sensory stimuli during swimming can either affect the network or not, depending on the precise timing of the stimulus in the swimming cycle (Figs. 16 and 17).

Once we had established that the model neuron and network responses were very close to those recorded in the young tadpole, we were able to test whether the type-specific properties of spinal neurons were actually important for network function during swimming. We focussed on dINs which excite all CPG neurons on their own side. Replacing singlespike firing dINs with neurons that fire repetitively leads to instability of the swimming behaviour and the production of synchronous motor output on both sides. Replacing them with delayed firing cINs leads to a complete breakdown in 
swimming activity. This shows that differences in properties between neurons can be significant for the operation of a network and that, in this case, the type-specific properties of dINs are important for swimming. These properties provide one mechanism for rhythm generation in swimming (see also Roberts and Tunstall, 1990) because they will fire again during depolarisation when they receive mid-cycle inhibition which hyperpolarises the membrane and leads to delayed firing on rebound. The model dINs have this property and there is now direct physiological evidence for it from dIN recordings (Li et al., 2006). Furthermore, if all neurons in the network are given the properties of dINs, swimming is nearly as stable as the control network over a range of strengths of synaptic excitation and inhibition (Fig. 14).

If firing a single spike to current and showing postinhibitory rebound is central to the generation of swimming, why are many of the spinal neurons able to fire repetitively when modelling suggests that giving them the ability to fire only once has almost no impact on the swimming rhythm? A possible explanation is that the spinal network must also generate the struggling pattern of motor output where neurons fire a high frequency burst on each cycle of a slower rhythm. Recordings have shown that motoneurons and some inhibitory interneurons are active during both activities (Soffe, 1993). During swimming, if driven by brief excitation from dINs, these neurons will only fire single spikes, but during struggling, with slower, more sustained excitation, they have the capacity to fire fast and repetitively in bursts. Other repetitively firing interneurons, the inhibitory aINs, usually fire a single spike on each swimming cycle but can sometimes fire twice and are vigorously active during struggling (Li et al., 2002). Finally, some repetitively firing interneurons that are not active during swimming become active and fire bursts during repetitive skin stimulation that leads to struggling. The role of repetitively firing neurons in the struggling network is the subject of current physiological and modelling studies ( $\mathrm{Li}$ et al., in prep.). The differences in firing properties may partly be a consequence of the need to switch rapidly between struggling and swimming, which may not make modulation of firing properties a practical alternative (Green and Soffe, 1996).

These observations, based on the young tadpole spinal cord, show how type-specific neuronal properties can be significant in network operation. They also indicate how this significance may not be fully apparent unless the whole range of potential network activity is taken into account; in the case of the tadpole, the requirement to struggle as well as swim. The properties of excitatory dINs appear to be specialized for swimming, showing only a single spike to current and post-inhibitory rebound. Other neurons cannot be substituted for dINs in the swimming CPG network. The specialised properties of dINs contribute to a rhythm genera- tion process for swimming based on feedback excitation and post-inhibitory rebound ( $\mathrm{Li}$ et al., 2006), but we know that this is not the whole story. There must be other rhythm generating processes operating in the tadpole CNS as a single side can generate rhythm (Soffe, 1989) as has recently also been shown in the lamprey (Cangiano and Grillner, 2005). The idea that recurrent inhibition within a single side (from aINs) could play a role in such rhythmic activity has already been tested (Roberts and Tunstall, 1990). Finally, the significance of the other firing patterns shown by tadpole spinal neurons is not yet clear, but it is striking that the firing patterns of small mammal dorsal horn interneurons can be grouped into four very similar categories (Prescott and De Koninck, 2002). Do these firing patterns provide a basic palette of neuron types that can be specified in building spinal circuits?

\section{Appendix: The individual neuron parameters}

Input resistance and resting potential in all neuron types were matched by setting the leak conductance and leak reversal potential of their models. In all model neurons, we had to modify the maximum conductances of the ion-channels to match recorded responses.

\section{$a I N$}

Starting from Dale's parameters (Dale, 1995b), several changes were needed to fit our measurements. The Dparameters to all currents were decreased, to increase the action potential voltage threshold. To increase the spike frequency to depolarizing current, the time constants $\tau$ for the Na-activation and -inactivation were changed: $\tau$ for the activation $\left(\mathrm{m}_{\mathrm{Na}}\right)$ was decreased by increasing the A-parameter of its $\beta$ rate constant, and even more at high voltages by decreasing the C-parameter of the $\alpha$ rate constant; $\tau$ for the inactivation $\left(\mathrm{h}_{\mathrm{Na}}\right)$ was increased by decreasing the $\mathrm{A}$-parameter of its $\alpha$ rate constant, but restored at high voltages by decreasing the $\mathrm{C}$-parameter of its $\beta$ rate constant. The $\mathrm{D}$-parameters of the Na-current were decreased more than those of the $\mathrm{K}$-currents, to lower the threshold current and time constant further. To lower the time constant of Na-activation around threshold and zero voltage (i.e. to get shorter spikes), its Eparameter was decreased, and to increase the time constant of the fast $\mathrm{K}$-activation in that range, its E-parameter was increased. The maximum activation of the fast K-channels was increased, and its time constant decreased, by increasing the A-parameter of its $\beta$ rate constant. The time constant of the slow K-current was decreased, but it plays a less important role, as its maximum conductance is relatively low. 


\section{$M N$}

These neurons are very similar to aINs. To match the threshold behaviour (with the changed resting potential), the Dparameters of all currents were changed, and the activation was sped up by increasing the A-parameter of the $\alpha$ rate constant of the Na-activation. All maximum conductances were decreased, to match threshold current and voltage. The E-parameter of the $\alpha$ rate constant was increased again to the value used by Dale (1995b). The A-parameter of the $\beta$ rate constant of $K_{\text {fast }}$ was decreased to get a deeper AHP and the A-parameter of the $\beta$ rate constant of the Na-inactivation was increased. To reduce spike width, the D-parameters of the Na-inactivation were increased more than others, which results in a lower time constant.

\section{$d I N$}

A unique property of dINs is that they never fire repetitively to sustained current, no matter how strong it is. To match this, starting from the aIN parameters, we increased the Cparameters of the $\alpha$ rate constant of Na-activation $\left(\mathrm{m}_{\mathrm{Na}}\right.$; from 1 to 4 ), of the $\beta$ rate constants of Na-inactivation ( $\mathrm{h}_{\mathrm{Na}}$, and of the slow K-current. In addition, the maximum conductance of slow $\mathrm{K}$ was increased, and that of fast $\mathrm{K}$ was decreased. To match the change in firing threshold, and to compensate for the big difference in input resistance (compared to aINs), all D-parameters were increased. To match the threshold, the D-parameters of the Na-currents were changed more than the other D-parameters. The parameters for the $\beta$ rate constant of the (less important) fast K-current were set to Dale's values. To match spike width and AHP, the A-parameters of the Na-inactivation were changed, which causes a larger time constant and a higher maximum value (i.e. wider spikes and larger impact).

\section{$R B$}

$\mathrm{RB}$ neurons have a rather negative resting potential. Starting from the dIN current parameters, most of the D-parameters were changed (except for the Na-activation) to get the right threshold voltage and current. The time constant of the sodium inactivation $\left(\mathrm{h}_{\mathrm{Na}}\right)$ was reduced, to reduce spike width, by decreasing its A-parameters. The maximum conductance of the slow K-current could be increased to suppress repetitive firing, and that of the Na-current decreased, which allowed the C-parameters of the $\alpha$ rate constant of Na-activation $\left(\mathrm{m}_{\mathrm{Na}}\right)$ to be reduced to 1 , as in Dale's model.

\section{$d l c$}

To get the adapting behaviour, we wanted the slow K-current to increase at each spike fired, and remain sufficiently ac- tivated that the neuron would not start to fire again after a while. To obtain this, the D-parameters of the slow $\mathrm{K}$ current were decreased for the $\alpha$ rate constant and increased for the $\beta$ rate constant. The A-parameters of these rate constants were increased and decreased respectively. The other D-parameters were decreased (compared to aIN) to match the threshold voltage and current. By increasing the C-parameter of the $\alpha$ rate constant of $\mathrm{m}_{\mathrm{Na}}$, the threshold for repetitive firing was raised. In this way, once repetitive firing starts, the frequency is higher. And it is specifically this high initial frequency that was needed. To the same effect, the $\mathrm{D}$-parameters of the fast K-current were decreased (more than the other current's D-parameters), and the maximum conductances of $\mathrm{Na}$ and fast K-currents were increased.

dla

To compensate for the much higher input resistance and lower threshold current, the conductances of all currents were decreased (starting from dlc parameter values). To then match the threshold voltage and current, all D-parameters were decreased slightly, and the one for the $\alpha$ rate constant of the Na-activation more than the others. To increase spike width, the A-parameters for Na-activation were decreased, and to get a low initial firing frequency, the C-parameters of the $\alpha$ rate constant of the Na-activation and of the $\beta$ rate constant of the fast K-current were decreased.

cIN :

The characteristic firing properties of cINs are repetitive firing following with a delay after an initial single spike. The maximum conductance for the Na-current was increased significantly (relative to the other currents) to overcome the higher leak conductance and get a high firing frequency. All current parameters (A, B, C, D and E) were kept similar to those for aINs. The maximum conductances of all currents were altered, to obtain the right firing threshold. The $\mathrm{C}$-parameter for the $\alpha$ rate constant of the Na-activation was decreased, which lowers the time constant for high voltage, resulting in narrow spikes and fast repetitive firing. To match spike width further, small changes were made to the A-parameters of the Na-inactivation. To increase the time constant of the fast $\mathrm{K}$-current, the $\mathrm{C}$-parameter of its $\beta$ rate constant was decreased.

The new K-current added to produce the delay in repetitive firing was initially given the same parameters as the Nacurrent, which also shows both activation and inactivation. The D-parameters were then decreased to match threshold and spiking behaviour, but the $\alpha$ rate constant for activation of the current $\left(\mathrm{m}_{\mathrm{A}}\right)$ was unaltered to maintain the pre-burst delay. The C-parameter for the inactivation rate constant $\beta$ was increased to 500: the higher this value, the longer the 
delay. The inactivation of the new current should hardly change when there is no injected current. To obtain this, the $\mathrm{C}$-parameter of its $\alpha$ rate constant is set to 1 , and the Aparameter very low. The A-parameter of its $\beta$ rate constant is set to 10 , to decrease the maximum opening rate. The Aparameters of the activation of the new current are high, so that it is fast enough to suppress immediate repetitive firing after the initial spike in response to sustained current. The slow inactivation of the new current will allow repetitive firing after a delay.

Acknowledgments Bart Sautois is a research assistant of the Research Foundation-Flanders (FWO_-Vlaanderen). The Bristol lab is supported by the Wellcome Trust.

\section{References}

Aiken SP, Kuenzi FM, Dale N (2003) Xenopus embryonic spinal neurons recorded in situ with patch-clamp electrodes-conditional oscillators after all? Eur. J. Neurosci. 18: 333-343.

Bem T, Le Feuvre Y, Rinzel J, Meyrand P (2005) Electrical coupling induces bistability of rhythms in networks of inhibitory spiking neurons. Eur. J. Neurosci. 22: 2661-2668.

Cangiano L, Grillner S (2005) Mechanisms of Rhythm Generation in a Spinal Locomotor Network Deprived of Crossed Connections: The Lamprey Hemicord. J. Neurosci. 25: 923-935.

Chapman DE, Keefe KA, Wilcox KS (2003) Evidence for functionally distinct synaptic NMDA receptors in ventromedial versus dorsolateral striatum. J. Neurophysiol. 89: 69-80.

Clarke JDW, Hayes BP, Hunt SP, Roberts A (1984) Sensory physiology, anatomy and immunohistochemistry of Rohon-Beard neurones in embryos of Xenopus laevis. J. Physiol. (Lond) 348: 511-525.

Dale N (1985) Reciprocal inhibitory interneurones in the Xenopus embryo spinal cord. J. Physiol. (Lond) 363: 61-70.

Dale N (1995a) Kinetic characterization of the voltage-gated currents possessed by Xenopus embryo spinal neurons. J. Physiol. 489(Pt2): 473-488.

Dale N (1995b) Experimentally derived model for the locomotor pattern generator in the Xenopus embryo. J. Physiol. 489(Pt2): 489-510.

Dale N (2003) Coordinated motor activity in simulated spinal networks emerges from simple biologically plausible rules of connectivity. J. Comput. Neurosci. 14: 55-70.

Dale N, Roberts A, Ottersen OP, Storm_Mathisen J (1987) The morphology and distribution of "Kolmer-Agduhr cells," a class of cerebrospinal-fluid-contacting neurons revealed in the frog embryo spinal cord by GABA immunocytochemistry. Proc. R. Soc. Lond. B. Biol. Sci. 232: 193-203.

Galarreta M, Hestrin S (1997) Properties of GABA(A) receptors underlying inhibitory synaptic currents in neocortical pyramidal neurons. J. Neurosci. 17: 7220-7227.

Gerstner W, Kistler W (2002) Spiking Neuron Models. Cambridge University Press.

Gotz T, Kraushaar U, Geiger J, Lubke J, Berger T, Jonas P (1997) Functional properties of AMPA and NMDA receptors expressed in identified types of basal ganglia neurons. J. Neurosci. 17: 204 215.

Green CS, Soffe SR (1996) Transitions between two different motor patterns in Xenopus embryos. J. Comp. Physiol. 178: 279- 291.

Grillner S, Wallen P (2002) Cellular bases of a vertebrate locomotor system-steering, intersegmental and segmental co-ordination and sensory control. Brain Res. Rev. 40: 92-106.
Hellgren J, Grillner S, Lansner A (1992) Computer simulation of the segmental neural network generating locomotion in lamprey by using populations of network interneurons. Biol. Cybern. 68:1.

Higashijima SI, Mandel G, Fetcho JR (2004a) Distribution of prospective glutamatergic, glycinergic, and GABAergic neurons in embryonic and larval zebrafish. J. Comp. Neurol. 480: 1-18.

Higashijima SI, Schaefer M, Fetcho JR (2004b) Neurotransmitter properties of spinal interneurons in embryonic and larval zebrafish. J. Comp. Neurol. 480: 19-37.

Hodgkin AL, Huxley AF (1952) A quantitative description of membrane current and its application to conduction and excitation in nerve. J. Physiol. (Lond) 117: 500-544.

Jezzini SH, Hill AAV, Kuzyk P, Calabrese RL (2004) Detailed model of intersegmental coordination in the timing network of the leech heartbeat central pattern generator. J. Neurophysiol. 91: 958-977.

Kahn JA, Roberts A, Kashin SM (1982) The neuromuscular basis of swimming movements in embryos of the amphibian Xenopus laevis. J. Exp. Biol. 99: 175-184.

Kotaleski JH, Grillner S, Lansner A (1999) Neural mechanisms potentially contributing to the intersegmental phase lag in lamprey. Biol. Cybern. 81: 317.

Li W-C, Perrins R, Soffe SR, Yoshida M, Walford A, Roberts A (2001) Defining classes of spinal interneuron and their axonal projections in hatchling Xenopus laevis tadpoles. J. Comp. Neurol. 441: 248265.

Li W-C, Soffe SR, Roberts A (2002) Spinal inhibitory neurons that modulate cutaneous sensory pathways during locomotion in a simple vertebrate. J. Neurosci. 22: 10924-10934.

Li W-C, Soffe SR, Roberts A (2003) The spinal interneurons and properties of glutamatergic synapses in a primitive vertebrate cutaneous flexion reflex. J. Neurosci. 23: 9068-9077.

Li W-C, Soffe SR, Roberts A (2004a) Glutamate and acetylcholine corelease at developing synapses. PNAS 101: 15488-15493.

Li W-C, Soffe SR, Roberts A (2004b) Dorsal Spinal Interneurons Forming a Primitive, Cutaneous Sensory Pathway. J. Neurophysiol. 92: 895-904.

Li W-C, Soffe SR, Roberts A (2004c) A direct comparison of whole cell patch and sharp electrodes by simultaneous recording from single spinal neurons in frog tadpoles. J. Neurophysiol. 92: 380-386.

Li W-C, Higashijima S, Parry DM, Roberts A, Soffe SR (2004d) Primitive roles for inhibitory interneurons in developing frog spinal cord. J. Neurosci. 24: 5840-5848.

Li W-C, Soffe SR, Wolf E, Roberts A (2006) Persistent Responses to Brief Stimuli: Feedback Excitation among Brainstem Neurons. J. Neurosci. 26: 4026-4035.

Lisman JE, Fellous JM, Wang XJ (1998) A role for NMDA-receptor channels in working memory. Nat. Neurosci. 1: 273-275.

Nieuwkoop PD, Faber J (1956) Normal tables of Xenopus laevis (Daudin). Amsterdam, North Holland.

Nowak L, Bregestovski P, Ascher P, Herbet A, Prochiantz A (1984) Magnesium gates glutamate-activated channels in mouse central neurones. Nature 307: 462-465.

Parker D (2001) Spinal-cord plasticity: independent and interactive effects of neuromodulator and activity-dependent plasticity. Mol. Neurobiol. 22: 55-80.

Perrins R, Soffe SR (1996) Local effects of glycinergic inhibition in the spinal cord motor systems for swimming in amphibian embryos. J. Neurophysiol. 76: 1025-1035.

Prescott SA, De Koninck Y (2002) Four cell types with distinctive membrane properties and morphologies in lamina I of the spinal dorsal horn of the adult rat. J. Physiol. (Lond) 539: 817-836.

Ribera AB, Nguyen DA (1993) Primary sensory neurons express a Shaker-like potassium channel gene. J. Neurosci. 13: 4988-4996.

Roberts A (2000) Early functional organization of spinal neurons in developing lower vertebrates. Brain Res. Bull. 53: 585-593. 
Roberts A, Dale N, Evoy WH, Soffe SR (1985) Synaptic potentials in motoneurons during fictive swimming in spinal Xenopus embryos. J. Neurophysiol. 54: 1-10.

Roberts A, Tunstall MJ (1990) Mutual Re-excitation with PostInhibitory Rebound: A Simulation Study on the Mechanisms for Locomotor Rhythm Generation in the Spinal Cord of Xenopus Embryos. Eur. J. Neurosci. 2: 11-23.

Roberts A, Walford A, Soffe SR, Yoshida M (1999) Motoneurons of the axial swimming muscles in hatchling Xenopus tadpoles: features, distribution, and central synapses. J. Comp. Neurol. 411: 472-486.

Sillar KT, Roberts A (1988) A neuronal mechanism for sensory gating during locomotion in a vertebrate. Nature 331: 262-265.

Sillar K, Roberts A (1990) Characterisation and function of spinal excitatory interneurons with commissural projections in Xenopus laevis embryos. Eur. J. Neurosci. 2: 1051-1062.

Soffe SR (1989) Roles of Glycinergic Inhibition and N-Methyl-DAspartate Receptor Mediated Excitation in the Locomotor Rhythmicity of One Half of the Xenopus Embryo Central Nervous System. Eur. J. Neurosci. 1: 561-571.

Soffe SR (1993) Two distinct rhythmic motor patterns are driven by common premotor and motor neurons in a simple vertebrate spinal cord. J. Neurosci. 13: 4456-4469.
Soffe S, Roberts A (1982) Activity of myotomal motoneurons during fictive swimming in frog embryos. J. Neurophysiol. 48: 12741288.

Soffe SR, Roberts A (1989) The influence of magnesium ions on the NMDA mediated responses of ventral rhythmic neurons in the spinal cord of Xenopus embryos. Eur. J. Neurosci. 1: $507-515$.

Soffe SR, Zhao FY, Roberts A (2001) Functional projection distances of spinal interneurons mediating reciprocal inhibition during swimming in Xenopus tadpoles. Eur. J. Neurosci. 13: 617-627.

Tunstall MJ, Roberts A, Soffe SR (2002) Modelling inter-segmental coordination of neuronal oscillators: synaptic mechanisms for unidirectional coupling during swimming in Xenopus tadpoles. J. Comput. Neurosci. 13: 143-158.

Wolf E, Zhao FY, Roberts A (1998) Non-linear summation of excitatory synaptic inputs to small neurones: a case study in spinal motoneurones of the young Xenopus tadpole. J. Physiol. 511(Pt3): $871-886$

Zhao FY, Burton BG, Wolf E, Roberts A (1998) Asymmetries in sensory pathways from skin to motoneurons on each side of the body determine the direction of an avoidance response in hatchling Xenopus tadpoles. J. Physiol. (Lond) 506: 471-487. 